\title{
The Proceedings of the International Conference
}

on Creationism

Volume 8

Print Reference: Pages 200-216

Article 8

2018

\section{Adam and Eve, Designed Diversity, and Allele Frequencies}

John C. Sanford

FMS Foundation

Robert W. Carter

FMS Foundation

Wes Brewer

Fluid Physics International

Follow this and additional works at: https://digitalcommons.cedarville.edu/icc_proceedings Inhn Baumgardner

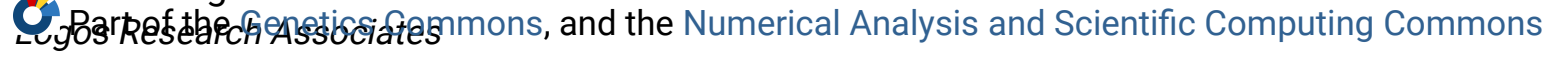

Bruce Rotter
B
FMS Foundations@Cedarville provides a publication platform for fully open access journals, which means that all articles are available on the Internet to all users immediately upon

publication. However, the opinions and sentiments expressed by the authors of articles

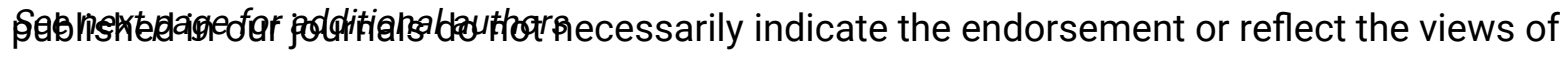
DigitalCommons@Cedarville, the Centennial Library, or Cedarville University and its employees. The authors are solely responsible for the content of their work. Please address questions to dc@cedarville.edu.

Browse the contents of this volume of The Proceedings of the International Conference on Creationism.

\section{Recommended Citation}

Sanford, J., R. Carter, W. Brewer, J. Baumgardner, B. Potter, and J. Potter. 2018. Adam and Eve, designed diversity, and allele frequencies. In Proceedings of the Eighth International Conference on Creationism, ed. J.H. Whitmore, pp. 200-216. Pittsburgh, Pennsylvania: Creation Science Fellowship. 


\section{Adam and Eve, Designed Diversity, and Allele Frequencies}

\section{Authors}

John C. Sanford, Robert W. Carter, Wes Brewer, John Baumgardner, Bruce Potter, and Jon Potter 


\title{
ADAM AND EVE, DESIGNED DIVERSITY, AND ALLELE FREQUENCIES
}

\author{
John Sanford, FMS Foundation, 877 Marshall Rd, Waterloo, NY 13165, USA. jsanford@FMSFound.org \\ Rob Carter, FMS Foundation, 877 Marshall Rd, Waterloo, NY 13165, USA. rcarter@FMSFound.org \\ Wes Brewer, Computational Solutions LLC, P.O. Box 4752, Jackson, MS 39296, USA. wes@computational.io \\ John Baumgardner, Logos Research Associates, 24515 Novato Place, Ramona, CA 92065 USA. Johnrbaumgardner@gmail.com \\ Bruce Potter, FMS Foundation, 877 Marshall Rd, Waterloo, NY 13165, USA. brucemp7@gmail.com \\ Jon Potter, FMS Foundation, 877 Marshall Rd, Waterloo, NY 13165, USA.jpotterm@gmail.com
}

\section{ABSTRACT}

Theistic evolutionists present multiple genetic arguments against a literal Adam and Eve. One key argument asserts it would be impossible for a single human couple to give rise to the genetic diversity seen in the modern human population. This implicitly assumes Adam and Eve would have been created without internal genetic diversity. If this were true, all observed variations would have to arise recently via random mutations. This would require incredibly high mutation rates, logically leading to rapid extinction.

Yet, Adam and Eve could have been created massively heterozygous. We have argued for over a decade that they could have been created with "designed diversity". We have previously shown that a vast amount of genetic variation could have been pre-programmed into their genomes. This could logically provide the genetic basis for: 1) our human gifts and talents; 2) the many forms of human beauty; and 3) the various ways people have rapidly adapted to new habitats.

It is also claimed that the currently observed human allele frequency patterns could not arise from a single couple. The logic here is that, since there were only four sets of chromosomes in Eden, all variants would have had an initial frequency of either $25 \%, 50 \%$, or $75 \%$. Today, most allelic variants have frequencies in the range of $0-10 \%$. Therefore, it is claimed that observed human diversity disproves a literal Adam and Eve.

In this paper we have critically examined these arguments. Our analyses highlight several genetic mechanisms that can help reconcile a literal Adam and Eve with the human allele frequency distributions seen today. We use numerical simulation to show that two people, if they contain designed alleles, can in fact give rise to allele frequency distributions of the very same type as are now seen in modern man.

We cannot know how God created Adam and Eve, nor exactly how Adam and Eve gave rise to the current human population. However, the genetic argument that there is no way that a literal Adam and Eve could have given rise to the observed human allele frequencies is clearly over-reaching and appears to be theologically reckless. There is no compelling reason to reject Adam and Eve based on modern allele frequencies.

\section{KEY WORDS}

human origins, demographic stirring, genetics, mutation, genetic drift, population bottleneck, designed diversity, designed alleles, designed gametes, founder effects, allele frequency distribution, numerical simulation.

\section{INTRODUCTION}

The attack on the historicity of Adam and Eve began long ago (VanDoodewaard 2015; Carter 2015) and has increasingly been coming from within the church (Venema and McKnight 2017; Carter 2017). Many theistic evolutionists are aggressively advancing the argument that Adam and Eve never existed, and so they must be either mythical or allegorical (Faulk 2004). They typically assert that there was no miraculous creation, no Edenic state, and no literal Fall.

Perhaps the most popular science-based argument against a literal Adam and Eve is the claim that it would be impossible for just two people to give rise to all the genetic diversity we see in the human population today. Some theistic evolutionists have been aggressively promoting this claim (e.g., Venema 2010; Venema and McKnight 2017). On various forums and blogs, some are even claiming they can prove the human population has never been less than several thousand individuals, or that that human allele frequency distributions are proof against a literal Adam and Eve (e.g., Shaffner 2017a, 2017b). Interestingly, other evolutionists caution that allele frequency analysis does not justify making dogmatic historical inferences (Myers, Fefferman, and Patterson 2008; Terhorst and Song 2015; Harpak, Bhasker, and Pritchard 2016; Baharian and Gravel 2018). This does not mean that allele frequency data are useless, however, only that one must be cautious when trying to derive historical models from them.

We have been exploring the concept that Adam and Eve might have been created in a heterozygous state for more than a decade. We call this the Designed Diversity Model. Other creation authors have also been thinking along these lines. For example, the idea 
appears at least as far back as Woodmorappe (1996), but also more recently in Lightner (2016), Jeanson and Tomkins (2016), Wood and Francis (2016), and Jeanson and Tomkins (2017). Likewise, ID proponents are now examining the concept of designed genetic diversity, for example, Hössjer et al. (2016a, 2016b), and Gauger (2018). Our 2005 version of the numerical simulation program Mendel's Accountant (hereafter "Mendel") included an "initial contrasting alleles" (ICA) option that was intended to enable the study of created diversity in a human population. At that time, we understood that the first human couple could have been designed with millions of variable genetic sites.

Mendel is best understood as an accounting program. Just as a large corporation or government must faithfully track a vast number of financial transactions and then calculate gain or loss at many different levels, Mendel tracks all of the old and new alleles that exist in a population, accounts for enormous numbers of genetic transactions that take place over many generations, and finally tallies final outcomes on many different levels.

Some may dismiss numerical simulation as an arbitrary "black box". This is unfortunate because numerical simulation has been widely tested and has become a powerful tool in many fields of applied science. Furthermore, Mendel was developed by highlevel scientists with proven expertise in the numerical simulation of real-world phenomena, and it has been widely validated in both creationist and secular literature (Sanford et al. 2007a; Sanford et al. 2007b; Baumgardner et al. 2008; Sanford et al. 2008; Sanford and Nelson 2012; Baumgardner et al. 2013; Brewer et al. 2013a; Brewer et al. 2013b; Brewer et al. 2013c; Gibson et al. 2013; Nelson and Sanford 2013; Rupe and Sanford 2013; Sanford et al. 2013; Sanford et al. 2015).

It is true that there are bad or dishonest numerical simulations, even as there are bad or dishonest accountants. But this does not invalidate financial accounting in general, nor does it invalidate genetic accounting in general. On the contrary, valid financial accounting can and must happen, and valid genetic accounting can and should happen.

The Designed Diversity Model requires an expanded vocabulary. Traditionally it has been assumed that genetic variation only comes from mutations, giving rise to mutational variants ("mutational alleles"). However, given a miraculous creation, there could be a very different class of created variants ("designed alleles"). Mutational alleles and designed alleles would be different in several important respects. Mutational alleles need time to accumulate, while designed alleles can exist from the beginning. Mutational alleles are essentially random typographical errors in the genome and so are typically harmful, while designed alleles would logically be created to be beneficial. While mutational alleles always arise in a population as a single isolated copy, designed alleles would logically be created at higher frequencies.

It is widely understood that a mutational allele arises as a single copy - which is, therefore, on the verge of its own extinction. When a new mutation enters a population, its frequency is just one copy in a population of $2 n$ (with $n$ being the population's size). Therefore, most mutational alleles are rapidly lost due to genetic drift within just a few generations (Rupe and Sanford 2013). While mutational alleles are typically very rare, designed alleles would typically be expected to be abundant, in accord with the nature of their function, and in accord with their initially designed frequencies.

The smallest possible unit of genetic variation involves a single letter difference in the genome. Population geneticists call these single nucleotide variants (SNVs). If the minor allele is found at a frequency greater than $1 \%$, such a variant allele is also called a single nucleotide polymorphism (SNP). For simplicity, and in keeping with the final report from the 1000 Genomes Project (1000 Genomes 2015), we will use the term "SNP" for all single nucleotide variations, regardless of their allelic frequency.

The 1000 Genomes Project detected 84 million SNPs within the human population (1000 Genomes 2015). The vast majority of these are very rare alleles (about 64 million of the observed SNPs had allele frequencies of less than $0.5 \%$ ). However, this is still a serious underestimate of how many rare human alleles exist. Given our current population size and mutation rate, every nucleotide site in the human genome should mutate many times every generation somewhere on this planet. Therefore, the number of existing SNPs should be roughly the size of the genome (3 billion). But most of these variants are so rare that they are not detectable, due to limited sampling size. Most rare human alleles are unique to a single people group or sub-population. This indicates that most of these rare variants have arisen via mutation in the relatively recent past.

We have previously proposed that, excluding rare alleles, a large fraction of currently observed human genetic diversity might have arisen from designed genetic variants that were built into Adam and Eve when they were first created (Sanford and Carter 2015a, $2015 b)$. The latest analysis of the human genome (1000 Genomes 2015), indicates that there are only 8 million SNPs with allele frequencies of $5 \%$ or more. Hypothetically, most of these common alleles could be designed alleles.

The average person living today carries 4-5 million SNP alleles (Levy et al. 2007). Therefore, a single human today accounts for a large fraction (approximately 30\%) of all common genetic variation (Carter 2018). The African people groups tend to have slightly higher rates of polymorphism (Gurdasani et al. 2014; 1000 Genomes 2015). Since there are only about 8 million common SNPs in the human population, and since most of the SNPs in a single person are common SNPs, this means that any given person carries a very significant percentage of all the common genetic variants found across the world (Carter 2018). A single modern couple should carry most of the 8 million common SNPs that are ubiquitous in the human population. Obviously, the genomes of Adam and Eve could have contained this amount of diversity and much more (Sanford and Carter 2015a, 2015b).

Some fraction of the pre-Flood genetic diversity would be lost due to the genetic bottleneck of the biblical Flood. However, population geneticists have known for decades that even the most extreme bottleneck (i.e., two people) can capture a significant amount of a population's pre-bottleneck diversity, assuming the bottleneck only lasts for one or just a few generations and is followed by rapid population re-growth (Nei et al. 1975). This has also been demonstrated using computer simulations of a singlegeneration Flood-type bottleneck involving just three founding 
couples (Carter and Powell 2016). Therefore, there is no problem with the Flood scenario in terms of preserving most of the originally designed variants, even though there would be some loss of diversity. For example, if Noah's three daughters-in-law were distantly related, the Ark-borne population could have carried up to $80 \%$ of the pre-Flood diversity (Carter and Powell 2016). Even in a worst-case scenario (where Shem, Ham and Japheth married their sisters), nearly $60 \%$ of the pre-Flood diversity would still have been retained (Carter 2018). Thus, while some created diversity would be lost at the Flood, Noah's family could have easily carried millions of polymorphic alleles.

In addition to the 8 million common alleles (most of which may be designed alleles), the 1000 Genomes Project identified another 64 million rare SNPs (most of which can be assumed to be mutational alleles). How many generations would it take for 64 million mutations to accumulate? Given a mutation rate of roughly 100 mutations per person per generation, and assuming our current population of over 7 billion people, it would require less than one generation to accumulate 64 million mutations in the human population. Even for a human population of just 10,000, it would only take about 80 generations. While most new mutational alleles usually drift out of a population, the rate of loss of mutational alleles would be greatly reduced in a population that is continuously growing rapidly. In light of all this, the blanket claim, "There is no way Adam and Eve could have given rise to so much diversity," is not reasonable.

While Adam and Eve could clearly have given rise to the currently observed amount of human genetic diversity, a more technical objection can still be raised. It deals with the specific distribution of the variant alleles observed in the human population. The narrower claim becomes, "Adam and Eve could not possibly account for the specific patterns of allele frequencies that we see in the modern human population." This more technical objection is not easily dismissed and calls for careful consideration.

To address these challenges, we developed a modified version of the Mendel program (version 2.7.2), and also a completely redesigned version of Mendel ("Mendel-Go") written in the state-of-the-art computer language "Go". We included a new dynamic population size function, so that special experiments could be conducted where population size was continuously dynamic (changing). We improved older features that enabled such things as tracking initially created alleles, studying normal mutation accumulation, and examining the effects of small founder populations, mid-run population bottlenecks, and subsequent population re-growth. Modifications were made so that the changing allele frequencies in the dynamic population could still be tracked across generations. At the end of each experiment, the final allele frequency distribution could be plotted and could be compared to actual allele frequency distributions seen in today's human population.

In this paper we will use logic and numerical simulation to show that the claim that "there is no possible way..." is overreaching. There are multiple genetic mechanisms that can reconcile the biblical Adam and Eve with the observed human allele distribution data.

\section{METHODS}

Our working hypothesis is that God miraculously created Adam and Eve with a vast amount of internal genetic diversity, such that there were millions of designed SNPs in Eden. We have used simple logic and numerical simulations to examine genetic mechanisms whereby a miraculously created first couple might give rise to an allele frequency distribution similar to that now seen in the human population.

\section{Plotting Actual Allele Frequency Distributions}

In order to observe the actual allele frequencies of the current human population, we employed the latest sequence data for the Y chromosome, the mitochondrial chromosome (see Diroma et al. 2014), and chromosome 22 sequence data from the 1000 Genomes Project page (accessed 17 Apr 2015). Allele frequency data were tabulated from the VCF-formatted data using custom Perl scripts. The data were plotted using standard Minor Allele Frequency (MAF) plots. These plots reflect the actual allele frequency distributions for the current human population. These plots are very informative in themselves and provide controls (templates) for comparisons with our numerical simulation results.

\section{Analysis of Theoretical Allelic Distributions Based Upon Numerical Simulations}

We tested various historical models and their expected allele frequency patterns using Mendel version 2.7.2 and Mendel-Go. As stated in the introduction, Mendel tracks the coming and going of virtual alleles that exist in a virtual population, accounts for enormous numbers of genetic transactions that take place over many generations, and tallies and plots final outcomes, including allele frequency distributions.

The modified Mendel program (version 2.7.2) required a new dynamic population size function, so that special experiments could be conducted where population size was dynamically changing. At the same time, an entirely restructured program (Mendel-Go) was developed. This was used to validate the output of the original Mendel simulator. These improvements enabled such things as initially created alleles, normal mutation accumulation, a small founder population, population growth, a population bottleneck, and population re-growth. Modifications were made so that the changing allele frequencies in the dynamic population could still be tracked across generations. At the end of the experiment, the final allele frequency distribution could be plotted and compared to actual allele frequency distributions seen in today's human population.

The model population grows each generation according to the following formula:

$$
p_{i+1}=\left\{\begin{array}{l}
\max \left(R_{A} \cdot p_{i}, P_{c}\right) \forall i \leq i_{b} \\
\max \left(R_{B} \cdot p_{i}, P_{c}\right) \forall i>i_{b}
\end{array}\right.
$$

where $i$ is the generation number, $i_{b}$ is the generation number when the bottleneck occurs, $R_{A}$ and $R_{B}$ are the average reproductive rates before and after the bottleneck, and $P_{c}$ is the carrying capacity of the population.

\section{Examining the Heterozygous Adam and Eve Model}

We tested the Heterozygous Adam and Eve Model using a series of numerical simulations. We used Mendel simulations to 
discover which parameters settings, if any, might allow a highly heterozygous first couple to generate allele distributions similar to the currently observed human allele frequency distributions. Since each one of the 88 original autosomes in Eden could have carried its own unique set of designed variants, normal chromosomal recombination and segregation could have generated a large number of genotypes in the second generation. In each succeeding generation allelic diversity would increase due to newly arising mutations and further recombination.

Our simulations required the creation and tracking of two very different types of genetic variation. The first type was the classically understood mutational allele, and the second type was the designed allele. Mutational alleles would arise essentially as word-processing errors in the genome. This type of mutational allele would always arise as a rare variant. Mutations are always occurring, and mutation count per individual consistently increases in number. From its inception, Mendel has always tracked each new mutation and each mutational allele. To simulate newly arising mutational alleles, we only had to specify the population's mutation rate and the effect of each mutation on fitness. Our default mutation rate was 100 mutations per person per generation. Our default mutational effect was "near-zero" (i.e., there was essentially no selection happening, all mutational alleles would be drifting).

In addition to mutational alleles, we simulated initial genetic variants that were created as designed allelic pairs, wherein each allele in a pair had its own designed function. Designed allele pairs would be present at the beginning of a Mendel run. To simulate this model, we had to create within Mendel a new computational function which establishes and tracks designed alleles. This new function allows the specification of: a) the number of designed allele pairs and their locations; $b$ ) the ratio of the paired alleles (1:1 or 1:3); and c) the fitness effect per pair (pairs are normally given equal but opposite fitness effects). Under the heterozygous Adam and Eve model, there would be just four copies of each chromosome in Eden, and so every designed allele pair would have a ratio of either $50 / 50$ or $25 / 75$ (so all initial allele frequencies for the designed alleles would be either 0.25 or 0.50 or 0.75 ). For most experiments, the magnitude of the fitness effects was always "nearneutral" (no effective selection).

\section{Examining the Designed Gametes Model}

We examined the logical outcome that would arise if God individually designed each of the gametes (more accurately the gametogonia) within Eden, with each gamete (or gametogonium) potentially having its own unique genotype. We tested to see if this could possibly generate the allele frequencies observed today. The logic of this analysis is described in the Results section. We first explain that two designed people could have millions of individually designed gametogonia, and that these diverse gametogonia could represent a gene pool essentially equivalent to the gene pool of a large human population. We then illustrate this using numerical simulations. We initially simulated 50 offspring that carried designed alleles from a first couple, which would have been transmitted through 100 genetically independent gametes ( 50 sperm and 50 eggs). Mendel then tracked the initial designed alleles, plus accumulating mutational alleles, though a 200-generation biblical framework (including population growth, a 6-person bottleneck in generation 9, and re-growth up to a pre-set maximum population size).

\section{Complexities of plotting allele frequencies from simulations that include designed alleles}

As stated above, all new mutations begin as very rare alleles. However, following the standard convention, we do not normally plot alleles with a frequency less than $1.0 \%$. Although these rare alleles account for most of the allelic diversity, we tally, but do not plot the very rare alleles. Instead, in our allele frequency plots the first (left-most) bin tallies the number of alleles with a frequency of $1-2 \%$, the next bin tallies alleles with a frequency of $2-3 \%$, etc. There are numerous practical reasons for this: a) detection of very rare alleles in this first bin is very sensitive to sampling size and so can fluctuate wildly; b) this first bin incorporates all DNA sequencing errors; $c$ ) this first bin is usually so large that it severely distorts the scaling of any allele frequency plot. We will revisit the importance of this "invisible bin" in the Discussion section.

Another major data plotting issue involves the question of whether we should plot allele frequencies from $1 \%$ to $50 \%$, or allele frequencies from $1 \%$ to $99 \%$. It is normally assumed that all alleles arise via random mutations, so it is assumed that there is an "original" (ancestral) allele and a "mutant" (derived) allele. It is usually also assumed that the original allele is the one most frequently observed (the major allele), and that the mutant allele is rare (the minor allele). Thus, allele frequency plots normally only show the minor allele (i.e., only allele frequencies between $1 \%$ and $50 \%$ are plotted), while the major allele is simply assumed. In our simulations, we actually know which alleles are original and which are derived by mutation (when scientists look at real human allele frequency distribution data, they cannot know which allele was the "original" since allele frequencies change over time). The normal convention is that only the minor alleles are plotted. Since for every minor allele at frequency $f$ there exists a major allele at a frequency $1-f$, if the major allele distribution was also plotted, it would appear as a mirror image of the minor allele distribution, making it redundant. For designed allele experiments, we always tally all alleles, but usually only plot the minor alleles (1-50\%). Only in a few special cases do we plot all alleles (1-99\%).

When discussing designed alleles and their allele frequency distributions we again need to clarify our terminology. When we simulate designed alleles, we cannot realistically adopt the terms "ancestral" or "derived". Likewise, when we specify designed allele pairs, both of the contrasting alleles will often start with a frequency of $50 \%$, so we cannot initially define the "major" or the "minor" allele. However, genetic drift will quickly "break the tie", at which point we can empirically classify the less abundant allele as the minor allele (as genetic drift continues, the major and minor alleles can "flip" over time).

\section{Details of simulations of evolutionary populations}

We simulated evolutionary human populations where there were no designed alleles. We generally specify 1000 individuals, with 100 new mutations being added per individual per generation. Except where noted, we have made all mutations effectively neutral, as is commonly assumed. This is essential for longer runs over many generations, or the population will go extinct due to slightly 
deleterious mutations, long before the simulation is complete.

\section{Details of simulations of short-term populations with designed alleles}

For our designed allele simulations, each initial contrasting allele pair represents two alternative nucleotides at the same genetic locus. The sum of their allele frequencies must add up to 1.0, and both alleles will always remain in the same linkage block. We normally make all designed alleles co-dominant. We typically assign fitness effects according to a Weibull distribution, and specify the upper limit of total fitness benefit (i.e., the hypothetical fitness increase if every "beneficial" allele went to fixation). Lastly, we specify the fraction of individuals in the population who are initially heterozygous. If the fraction is 1.0, then both Adam and Eve are equally heterozygous and all designed alleles begin with frequency of $50 \%$. Alternatively, if the initial heterozygous fraction is set to 0.5, then either Adam or Eve would be heterozygous for all alleles, while the other would be homozygous. In this case, all designed alleles start at a frequency of either $25 \%$ or $75 \%$.

\section{RESULTS}

\section{Actual Allele Frequency Distributions}

The allele frequency distributions within the 1000 Genomes Project data for chr22, chrY, and chrM are shown in Figures 1a-c. Summary allele data for each of the three chromosomes are reported in Table 1. Figure 1a is the allele frequency distribution benchmark for this paper. At the time of submission, our analysis only included chr22 data. However, we have since calculated the allele frequency distribution for all human autosomal chromosomes included in the 1000 Genomes database and have observed a distribution essentially identical to chr22. Thus, in this case, chr22 is a suitable proxy for the rest of the genome.

\section{Illustrating the Evolutionary Model - simulations without designed alleles}

In our evolutionary simulations, we have observed that mutational allele frequency distributions are determined by the rate of genetic drift, which is dependent on the parameter settings for population size, mutation rate, and time. For any biologically realistic population, the number of accumulated mutations increases linearly with time. Mutational alleles continuously enter the population at very low initial frequencies and those that are not lost to drift will very slowly drift toward the right (i.e., away from zero). The rate of drift in any population with 1,000 or more individuals is exceedingly slow. Only after deep time can a large population reach mutation/drift equilibrium, where older alleles are drifting to fixation as fast as new alleles are drifting into the population. When mutation/drift equilibrium is reached, the allele frequency distribution stabilizes. At the same time, the total number of polymorphisms in the population stops increasing. An example of an allele frequency distribution of an evolutionary population in mutation/drift equilibrium is shown in Figure 2a. In this run the population size was 1000 , the mutation rate was 100 mutations per individual per generation, and it ran for 10,000 generations. Figure 2 a shows the full range of allele frequencies $(1-100 \%)$. As can be seen, a large number of alleles have drifted to fixation (100\%). Figure $2 \mathrm{~b}$ follows the convention of only plotting the minor allele frequencies $(1-50 \%)$. Figure $2 \mathrm{~b}$ is our benchmark for a stable

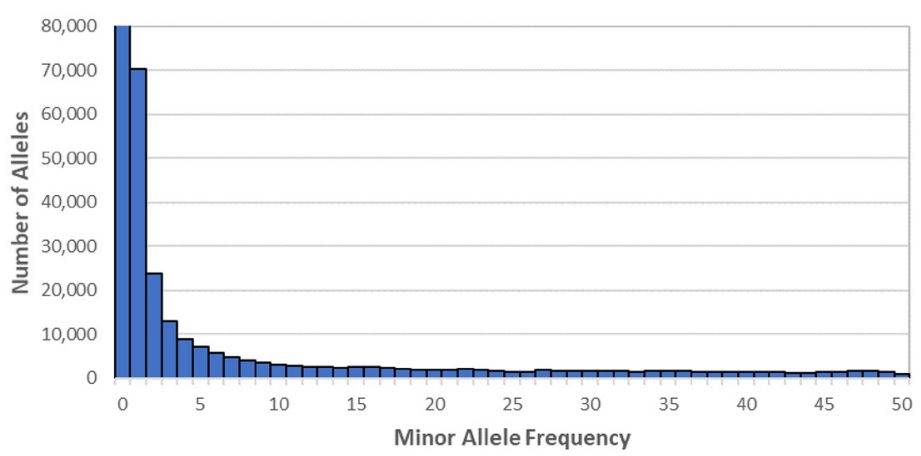

Figure 1a. The minor allele frequency distribution for human chromosome 22, based upon 2,504 individuals from the 1000 Genomes Project. The vast majority of SNPs in the first bin $(702,725)$ are not shown.

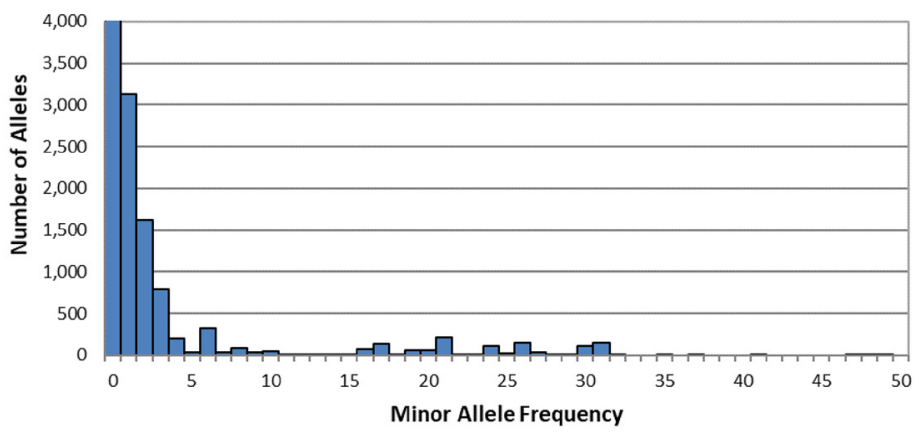

Figure 1b. The allele frequency distribution for the human chromosome Y, based upon 1,209 individuals from the 1000 Genomes Project. The vast majority of SNPs in the first bin $(52,955)$ are not shown. The extreme scarcity of high-frequency alleles suggests that chromosome $\mathrm{Y}$ is young.

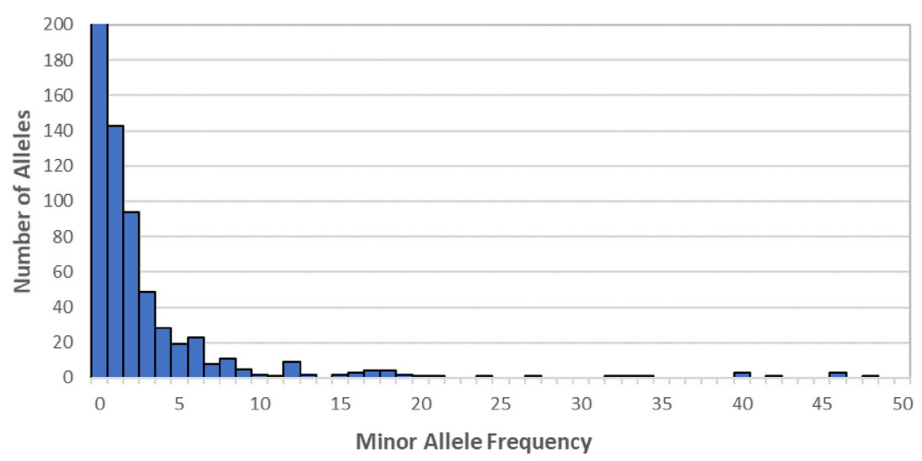

Figure 1c. The allele frequency distribution for the human mitochondrial chromosome, based upon 1,074 individuals from the 1000 Genomes Project. The vast majority of SNPs in the first bin $(2,194)$ are not shown. The extreme scarcity of high-frequency alleles suggests the mitochondrial chromosome is young.

Table 1. Three chromosomes, the number of sequenced individuals (n), the total number SNPs for each chromosome, and number of common SNPs for each chromosome, based on 1000 Genomes Project data.

\begin{tabular}{|c|c|c|c|}
\hline Chromosome & $\mathbf{n}$ & All SNPs & $\begin{array}{c}\text { Common } \\
\text { SNPs }\end{array}$ \\
\hline 22 & 2,504 & 918,038 & 215,313 \\
\hline Y & 1,233 & 60,446 & 7,491 \\
\hline MT & 1,074 & 2,618 & 424 \\
\hline
\end{tabular}


A
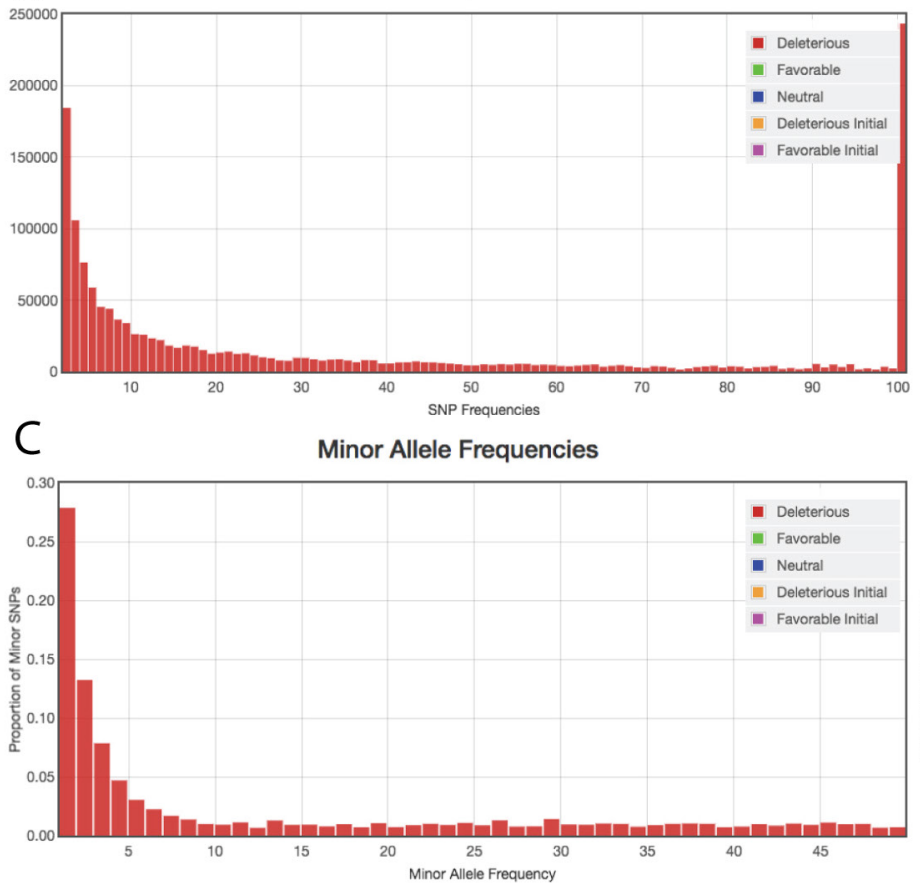

B Minor Allele Frequencies

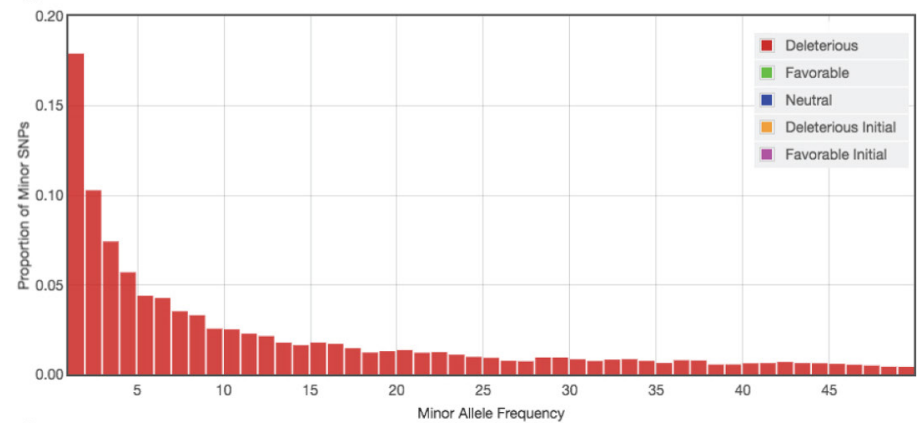

D

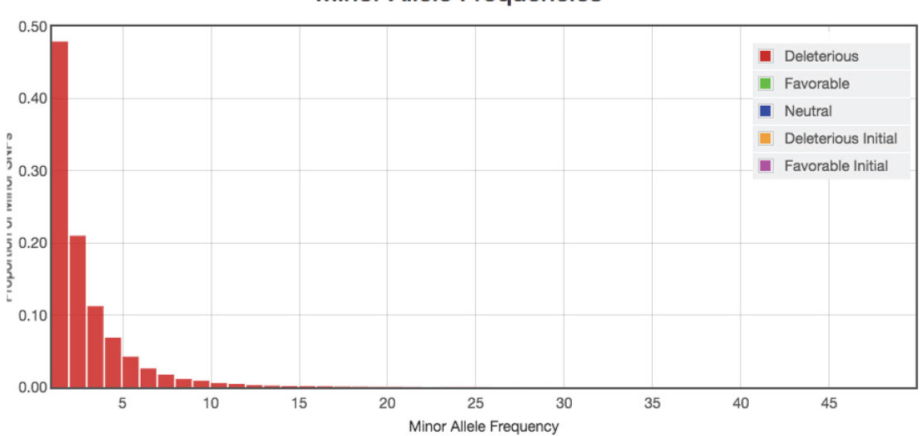

Figure 2a. Allele distribution of a simulated evolutionary population that is mature and is in mutation/drift equilibrium (case p0a098). There were no designed alleles. Key parameters settings were 10,000 generations; 1000 population size; 100 mutations per individual per generation. This figure plots allele frequencies from $1-100 \%$ to show that fixations (far right) are arising at a high rate, indicating that this population was in mutation/drift equilibrium. The same case is show in Figure 2b, but this plot shows the conventional plotting of only the minor alleles (1-50\% frequencies). Simulated and plotted using new Mendel-Go version.

Figure 2b. This figure shows the same evolutionary simulation as Figure 2a, but with only minor alleles are plotted (0-50\%). Case p0a098. Simulated and plotted using new Mendel-Go version.

Figure 2c. This is what can be considered an evolutionary Adam and Eve scenario. This Figure has the same setting as Figure $2 \mathrm{~b}$ but adds a severe single-generation bottleneck just 200 generations before the experiment ended (case i2e1e0). There were no designed alleles. Key parameters settings were 1000 population size; 10,000 generations; 100 mutations per individual per generation; a bottleneck at generation 9,800; and a re-growth rate of 1.5 after the bottleneck. The plotted allele frequencies are from 1-50\%. Simulated and plotted using new Mendel-Go version.

Figure 2d. This distribution reflects mutation accumulation in a biblical timeframe (case pc1fe3). There were no designed alleles. Key parameters settings were an initial population size of two, a stable population size of 1000; 200 generations; 100 mutations per individual per generation; a bottleneck at generation 10; and a population growth and re-growth rate of 2.0 (doubling every generation). The plotted allele frequencies are from $1-50 \%$. This plot is nothing like the actually observed autosomal allele distribution but is more similar to the actually observed chrY (Figure $1 \mathrm{~b}$ ) and chrM (Figure 1c) allele distributions. Simulated and plotted using new Mendel-Go version.

evolutionary allele distribution. Our simulation of this type of evolutionary population in deep time generates an allele frequency distribution that is quite different from the actually observed allele distributions from the 1000 Genomes Project (Figure 8, Table 2). The actually observed allele distribution has a distinctly tighter bend in its distribution, compared to the evolutionary scenario, in the frequency range of $3-15 \%$.

Figure 2c shows an identical simulation, except a bottleneck occurs 200 generations before the run is over. The population shrinks down to just two people for a single generation and then rapidly rebounds to its original size. This represents an Evolutionary Adam and Eve scenario, wherein Adam and Eve derive naturally from a larger human population and then give rise to modern humanity. As can be seen, the Evolutionary Adam and Eve scenario yields an allele distribution that is clearly similar to the actually observed distribution (Figure 1a) but is distinct from the evolutionary simulation (Figure 2b). See Figure 8 and Table 2. The bend in the curve is distinctly sharper in the Evolutionary Adam and Eve

\section{simulation.}

Figure $2 \mathrm{~d}$ shows a mutation accumulation simulation, but with a biblical timeframe and population dynamics. In this case the run starts with two individuals, there is rapid population growth for 10 generations, then there is a bottleneck down to 6 people, followed by rapid population rebound up to 1000 . The run only lasts 200 generations. In this biblical framework, we see substantial genetic drift leading to a meaningful allele distribution spread, even after just 200 generations. Yet the distribution in Figure $2 \mathrm{~d}$ does not look like the actually observed distribution of the autosomal chromosome 22 (Figure 1a). However, this simulation is quite consistent with the observed allele distributions of chrY (Figure 1b), and chrM (Figure 1c). This is not surprising from a creation perspective, because in Eden the autosomal chromosomes would initially exist in four copies (enabling initial heterozygosity), while there would be only one progenitor chrY and only one progenitor chrM. Therefore, it might be expected that the autosomes might carry designed heterozygosity, while chrY and chrM would initially 
Table 2. Differences between the normalized observed allele distribution (chromosome 22) and the normalized distributions of several models. The Mean Squared Error (MSE) is simply the average of the error terms $\left(Y_{i}\right.$ $\left.-\hat{Y}_{i}\right)^{2}$, where $Y_{i}$ is the value of the test distribution and $\hat{Y}_{i}$ is the value of the reference distribution (in this case, chromosome 22) at that same frequency. Smaller values indicate a tighter fit to the reference sequence. The MSE is not a significance test, but the results are a useful guide when tweaking parameters within a model to make the model fit an expected distribution. The most divergent model was the evolutionary simulation. The three Adam and Eve models all show a much tighter fit to chromosome 22. Further experimentation with parameter settings will improve the fit of the Designed Alleles and Designed Gametes models.

\begin{tabular}{|c|c|c|}
\hline Model & $\begin{array}{c}\text { Corresponding } \\
\text { Figure }\end{array}$ & MSE $\left(\mathbf{x 1 0}^{-3}\right)$ \\
\hline Evolutionary & $2 \mathrm{~b}$ & 5.75 \\
\hline $\begin{array}{c}\text { Evolutionary } \\
\text { Adam and Eve }\end{array}$ & $2 \mathrm{c}$ & 0.70 \\
\hline $\begin{array}{c}\text { A \& E, } \\
\text { Designed Alleles }\end{array}$ & $6 \mathrm{~b}$ & 1.16 \\
\hline $\begin{array}{c}\text { A \& E, } \\
\text { Designed Gametes }\end{array}$ & 7 & 0.84 \\
\hline
\end{tabular}

be invariant. Figure $2 \mathrm{~d}$, like Figures $1 \mathrm{~b}$ and $1 \mathrm{c}$, may all reflect non-variant Edenic chromosomes that lacked designed variants. This makes sense, because in all three cases there are very few SNP alleles, and their allele distributions are very strongly skewed toward lower allele frequencies. These distributions only make sense if these chromosomes were very young and were initially invariant. If all the autosomal chromosomes initially lacked designed variants, it seems possible that they might similarly appear very young.

\section{Illustrating the Heterozygous Adam and Eve Model}

Could the observed human allele frequency distributions be derived from a massively heterozygous Adam and Eve? We used numerical simulations to examine this question.

\section{A. Simulations involving only designed alleles, all having an initial frequency of $50 \%$.}

We first performed simulations with only designed alleles (no new mutations arising). Note that most of these experiments show only the minor allele but still show a full frequency range of $0-100 \%$. We initially specified that all alleles start with an allele frequency of $50 \%$. For these simulations we generally specified zero mutations, a short timeframe (200 generations), 989 designed allele pairs, 989 linkage blocks, and nearly-neutral fitness effects (i.e., no selection).

The initial allele frequency distribution (first generation) for all such runs is shown in Figure 3a. The allele "distribution" is a single spike at $50 \%$. Given this starting point, and given a constant population size of 1000, after 200 generations the distribution was a narrow bell-shaped curve that was still centered at $50 \%$, indicating that in this time there had been very limited genetic drift (Figure $3 b)$. A second, more biblically-realistic experiment involved the same initial allele frequency and a time span of 200 generations, but started with a population of two, followed by rapid population growth, a severe population bottleneck to just 6 people in the tenth
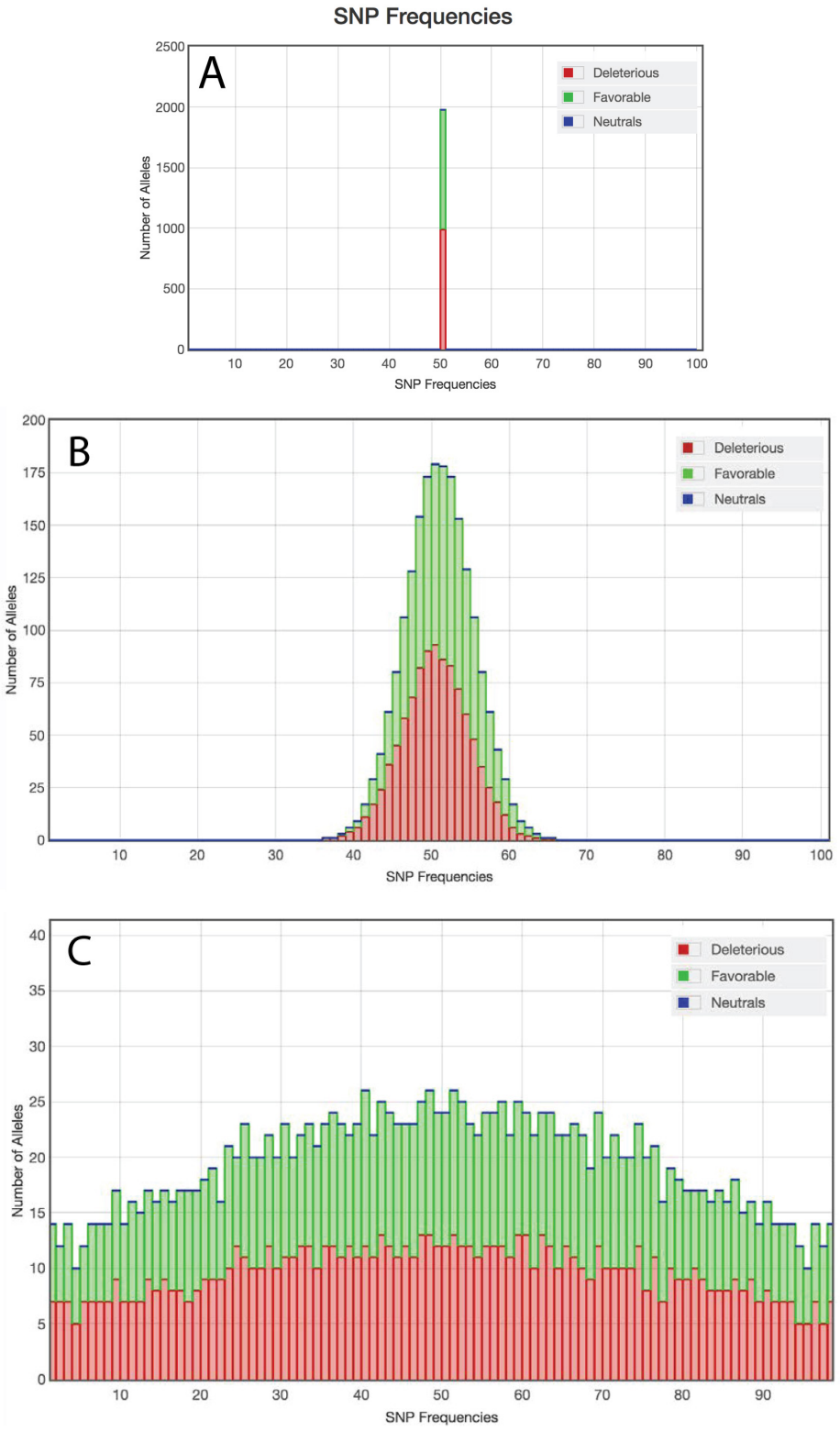

Figure 3a. A simple illustration of a designed allele "distribution."In the first generation of a simulation. In this example, all designed allele pairs begin with a 50/50 ratio, so all designed alleles in this experiment start with an initial frequency of $50 \%$. Because for each contrasting allele pair, one allele has one desireable function and one has another alternative desireable function, we arbitrarily designated one allele "beneficial" (green) and the other allele is arbitrarily designated "deleterious" (red). These alleles are made nearly neutral (unresponsive to selection). Note that in these cases Mendel has plotted allele frequencies from 1-100\%. Simulated and plotted using Mendel version 2.7.2. Figure 3b. This is a simple illustration of a designed allele distribution, similar to Figure 3a, but after 200 generations of drift. It assumes no mutations and a population with a constant population size of 1000. It is obvious that drift has happened, but in this timeframe the drift is modest. Mendel has plotted allele frequencies from 1-100\%. Simulated and plotted using Mendel version 2.7.2. Figure 3c. This is a simple illustration of a designed allele distribution, similar to Figure 3b, after 200 generations of drift, with no mutations, but with two severe population constrictions. The starting population size was 2 , followed by rapid growth and an extreme single-generation bottleneck in generation 10 (6 individuals), followed by rapid growth up to 1000 . As can be seen, biblical population dynamics greatly increase the rate of genetic drift. Mendel has plotted allele frequencies from $1-100 \%$. Simulated and plotted using Mendel version 2.7.2. 
generation, followed by a rapid population rebound (Figure 3c). In this biblical scenario, we see that two population constrictions can result in much more rapid allele frequency spreading.

\section{B. Simulations involving only designed alleles, all having an initial frequency of $25 \%$}

Still more promising were simulations that involved designed alleles that all began with a minor allele frequency of $25 \%$. For every allele pair where the minor allele frequency is $25 \%$, there is a contrasting allele with allele a frequency of $75 \%$ (we generally only show the minor alleles in such plots). In this case, the starting allele frequency distribution (in the first generation) would look just like Figure 3a, but with the spike located at $25 \%$. With this lower initial allele frequency, it is much easier to approach the observed human frequency distribution (Figure 1a). Figure 4a shows what happens after just 20 generations. Most of the distribution is shifting to the left, with the mode shifting downward from $25 \%$. Figure $4 \mathrm{~b}$ shows what happens after 200 generations. The mode is now approaching zero and the distribution's bell-shaped curve has become a nearly straight line that slopes downward to the right. When re-scaled, this is the basic shape of the lower-most portion of the actual allele frequency distribution. We have found that the steepness of the slope can be modulated by including in the simulation some fraction of allele pairs that start at $50 \%$. The steepness of the slope diminishes greatly when mutational alleles are added and begin to accumulate to high numbers (because this requires the y-axis of the frequency plots to be rescaled).

\section{Simulation involving high-impact designed alleles resulting in selective sweeps}

Figure 5 shows the effect of a series of selective sweeps. In these experiments a limited number of designed allele pairs (initially all at 50\%) were assigned large fitness effects and were simulated within a biblical framework. We show both the minor (red = less favored) and major (green $=$ more favored) alleles in this plot. These allele pairs responded rapidly to natural selection, effectively emptying the central part of the distribution and driving the high impact alleles to the left and right extremes (this effectively fills "gap zone" separating the high-frequency created alleles and lowfrequency mutational alleles). Such strong selective sweeps would be expected to carry many low-impact linked alleles along with them. In the Discussion section we will describe the implications of numerous strong demographic forces (such as selective sweeps), that can eclipse the effects of drift.

\section{Simulations combining both mutational alleles and designed alleles}

We did a preliminary simulation where we combined designed alleles (initially all at 50\%) with mutational alleles (Figure 6a). The resulting distribution reveals that the rare mutational alleles on the far left, and the designed alleles are spreading along the $\mathrm{x}$-axis, with the mode at $50 \%$. This outcome is obviously very different from the observed allele distribution.

We then did an experiment where designed alleles (initially all at $25 \%$ ) were combined with mutational alleles (Figure $6 \mathrm{~b}$ ). The resulting distribution reflects the relatively rare mutational alleles on the far left, smoothly transitioning into the designed alleles spread along the $\mathrm{x}$-axis. The resulting distribution was very similar
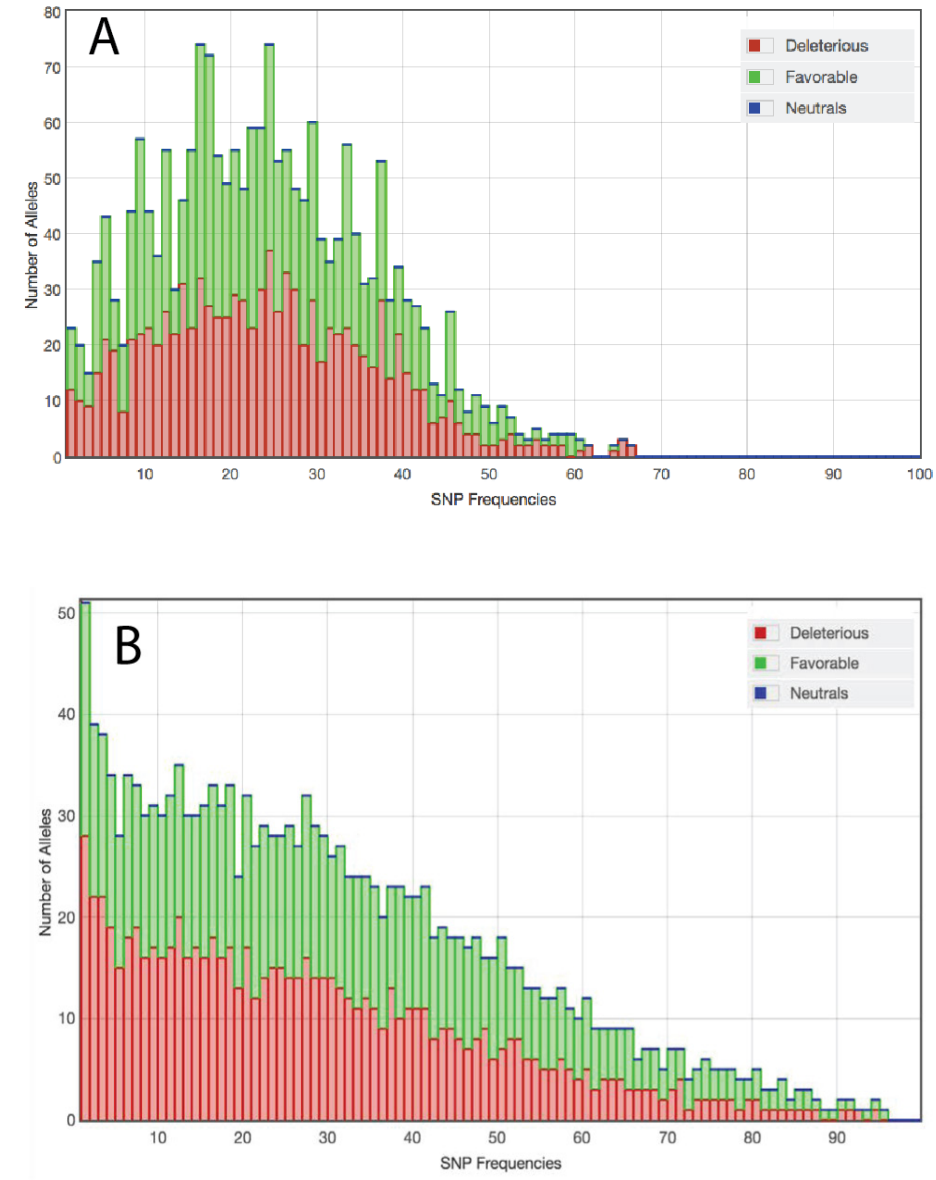

Figure 4a. This is a simple illustration of a designed allele distribution, similar to Figure 3b, but with all initial minor alleles starting at a frequency of $25 \%$, and after just 20 generations. The designed alleles have a distribution strongly skewed toward the left. Mendel has plotted allele frequencies from $1-100 \%$. Simulated and plotted using Mendel version 2.7.2.

Figure $4 \mathbf{b}$. This is a simple illustration of a designed allele distribution, similar to Figure $4 \mathrm{a}$, but after 200 generations of drift. It assumes no mutations and a population with a constant population size of 1000 . The designed alleles have a distribution strongly skewed toward the left. Instead of the "hump" that is observed in the middle frequency range as is seen when designed alleles start out with a frequency of $50 \%$ (Figure $3 \mathrm{c}$ ), this distribution is resembles a straight line sloping downward to the right. When mutations are added and the scale is normalized, this type of curve approximates the lower section of the actual allele frequency distribution. When biblical population constrictions are added there is much more allele spreading, resulting in a distribution that is nearly flat. Mendel has plotted allele frequencies from 1-100\%. Simulated and plotted using Mendel version 2.7.2.

to the actually observed allele distribution (Figure 8, Table 2). The exact shape of this curve would depend on the early population growth rates, as well as various population dynamics such as selective sweeps, lineage extinctions, etc., as will be discussed.

\section{Illustration of the Designed Gametes Model Using Logic and Numerical Simulation}

Lastly, we examined the feasibility that God designed a unique genotype for each gametogonium of Adam and Eve. Given the premise of a miraculously created Adam and Eve, a logical way for God to bless later generations with abundant "good" diversity 
would be to create within Adam and Eve genetically diverse gametogonia (the cells that give rise to gametes). Normally, a woman's egg cells form from her gametogonia while she is still in her mother's womb. In other words, women are normally born with a vast number of eggs already formed in their ovaries. However, assuming that Eve was created, not born, her eggs could not have formed in the normal way - so each gametogonium would have been miraculously formed and could potentially have been genetically unique. Therefore, there is almost no limit to the number of variant alleles and linkage blocks that could have existed in Eve's ovaries. Eve might have had a vast number of designed SNPs in each egg. Similar logic would apply to Adam's gametogonia (giving rise to sperm). In addition, all those designed gametic variants would logically have been created within linkage blocks that were designed, specific, and functional. Our Designed Gametes Model appears to not only help reconcile a literal Adam and Eve with observed allele frequencies, but also with observed linkage block patterns.

If there were individually designed gametes/gametogonia in Eden, this would potentially constitute an enormous gene poo1, comparable to the gene pool of a large human population. To transmit a large fraction of the original genetic diversity to later generations would require that the first family was very large. Indeed, it is entirely feasible that Adam and Eve would have had a very large family size, given the extreme longevity and vigor of the early patriarchs (Carter and Hardy 2015). In such an extremely large family, there could have been 100 or more different sets of chromosomes, representing a very substantial sampling of the primordial gene pool that existed within Adam and Eve's gametes. This means that the variants in that first human population could have started with almost any initial allele frequency distribution, in accord with God's design for mankind. In the same way, the initial population of gametes could have also started with a great diversity of linkage patterns, as might have been in accord with God's design.

We employed numerical simulations to illustrate how designed gametes might give rise to patterns of allele diversity similar to those observed today. To model designed diversity within gametes, we did not start our simulation with Adam and Eve, but with their children, because their children would represent a sampling of Adam and Eve's gametes. We assumed 50 children (100 chromosome sets). Each child had 989 linkage blocks, with each linkage block was assigned a specified number of designed allele pairs. We then simulated a biblical population that grew rapidly, experienced a severe bottleneck at generation 10 , and then grew rapidly again. The simulation was stopped after 200 generations, and the allele frequencies were tallied and plotted (Figure 7). As can be seen, both Figures 1a and Figure 7 yield very similar types of allele distributions (Figure 8, Table 2). However, the evolutionary simulation (Figure2b) shows a distribution which is distinctly different from what is actually observed and what our designed gametes simulation indicates. Figure 7 shows that the Designed Gametes Model enables a literal Adam and Eve to generate, in just 200 generations, and without any ancestors, an allele frequency distribution very similar to what is seen today. Mendel's parameter settings can obviously be fine-tuned to further

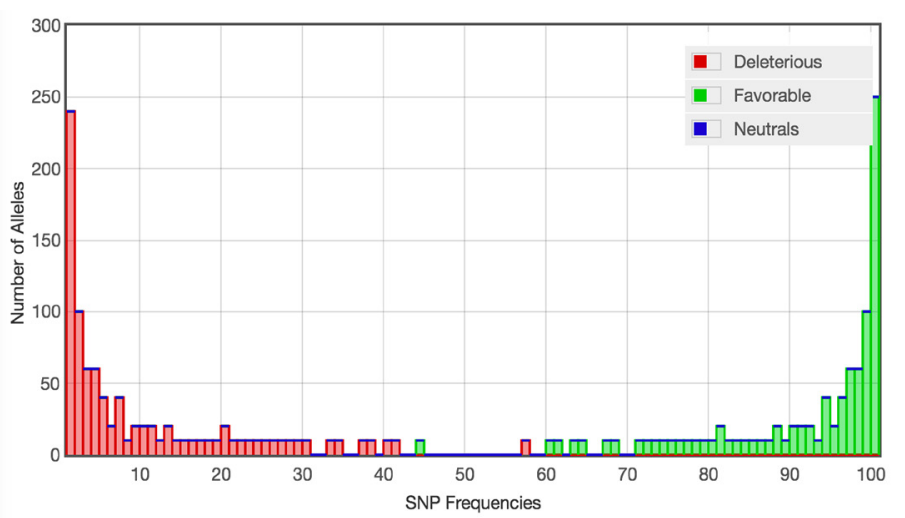

Figure 5. An illustration of high-impact designed alleles resulting in systematic selective sweeps. While Figures $3 \mathrm{~b}$ and $5 \mathrm{c}$ reflect the slow and diffusive nature of genetic drift, there are many other variables in nature that actively drive allele frequencies to change, and to change rapidly. Natural selection is one such force. This plot shows a limited number of designed allele pairs which are started with an initial frequency of $50 \%$ (as with Figure 3a). However, the designed allele pairs were assigned strong positive and negative fitness values which enabled strong natural selection. In just 200 generations, selection has completely separated the un-favored designed alleles (red) from the favored designed alleles (green). Selection has driven all alleles out of the center of the distribution. The un-favored alleles have been strongly pushed to the left, filling the problematic "gap" region (in the range of 3-15\%), that can arise where there is not a smooth transition between mutational allele distributions and designed allele distributions. Such high-impact designed alleles would sweep along with them a great number of nearly-neutral designed and mutational alleles that were linked to them. These "selective sweeps" drag many nearly-neutral alleles into the "gap zone". Note that in this case Mendel has plotted allele frequencies from $1-100 \%$. Simulated and plotted using Mendel version 2.7.2.

improve the match between our Designed Gametes Model and the actually observed allele frequency data. However, a very important caveat regarding Figure 7 and all our other results, is that the size of our simulated populations is usually just 1000 . This reason for this will be addressed in depth in the Discussion section.

\section{Comparing the Different Distributions}

Figure 8 helps us to compare the allele distributions of our five key simulations (Figures 1a, 2b, 2c, 6b, and 7). Figure 1a represents the allele distribution that is actually observed in human chromosome 22 (shown in black). Figure $2 \mathrm{~b}$ represents the allele distribution of a classical evolutionary simulation. Figure $2 \mathrm{c}$ represents the allele distribution of an evolutionary Adam and Eve simulation. Figure $6 \mathrm{~b}$ represents the allele distribution of a heterozygous Adam and Eve simulation. Figure 7 represents the allele distribution of an Adam and Eve simulation involving designed gametogonia.

Figure 8 displays these five normalized distributions side-by-side, for the purpose of comparison. As can be visually discerned, all of the curves that involve any type of Adam and Eve simulation are similar to each other and largely overlap with the actually observed distribution. However, the evolutionary simulation was most clearly divergent from the actually observed distribution. Table 2 shows the degree of difference of each curve, compared to the chr22 distribution. As can be seen, all three Adam and Eve simulations were very similar to the actually observed distribution, while the evolutionary simulation had a distribution that was by far 
the most divergent.

\section{DISCUSSION}

Any origin-of-man model will have significant problems due to the inherent limits of historical science. Thus, we suggest there should be a great deal of humility on all sides as we explore this very significant topic. Even as Bible believers, we should not pretend to understand the thoughts or actions of God, except as He has revealed them to us. The best we can do is to encourage the faithful by providing reasonable evidences and credible models that are consistent with the Word of God.

Before we address the various ways in which a literal Adam and Eve might be reconciled with the observed allele distributions, we must first point out that the human evolutionary model has many fundamental problems of its own. For example, there is strong evidence that human populations cannot survive in deep time due the relentless accumulation of slightly deleterious mutations (Sanford 2014). Many of the mutations that account for the millions of rare alleles in the human population should be very slightly deleterious. This should result in continuous genetic degeneration and eventual extinction. A second profound problem with the human evolutionary model is the fact that there is simply no credible way that mutation/selection can create the vast amount of new information that would be required to change an ape population into a human population. The enormous difficulty of creating the biological information that makes life, and makes us human, has been demonstrated on many levels (Marks et al. 2013). The counter-claim has been that the famous nylonase gene is proof that it is easy to create new functional biological information. However, the spontaneous nylonase claim has recently been falsified (Cordova and Sanford 2017). The human evolution model has a third glaring problem called "the waiting time problem". It turns out that it would take at least 84 million years to create and fix a nucleotide string consisting of only two letters in a human-like ancestral population (Sanford et al. 2015). Yet human evolution requires a vast number of specific nucleotide strings that are much longer than two letters long. A fourth serious problem associated with the human evolutionary model involves the fact that the bones that are popularly claimed to be "transitional fossils" are actually highly contested within the field of paleoanthropology (Rupe and Sanford 2017).

If we start with the premise of a miraculously created Adam and Eve, the idea of "designed diversity" is a logical deduction. It provides the most coherent explanation for the beneficial variations that we see within the human race today. Since all parties acknowledge that nearly all non-neutral mutations are deleterious, only designed variants can credibly account for all the "good diversity" we see (i.e., variations that are desirable, and have no pathological effects). This should be especially obvious when we consider the various forms of human beauty, and the various types of human gifts and talents such as mathematical or artistic genius. Desirable human variations of this type cannot rationally be attributed to Darwinian mutation/selection. In addition, when designed diversity is coupled with natural selection, it optimally enables rapid local adaptation and rapid fragmentation of populations into sub-populations after the Flood. It is reasonable to expect that diversity would be part of God's design, both for humans and for all living things.
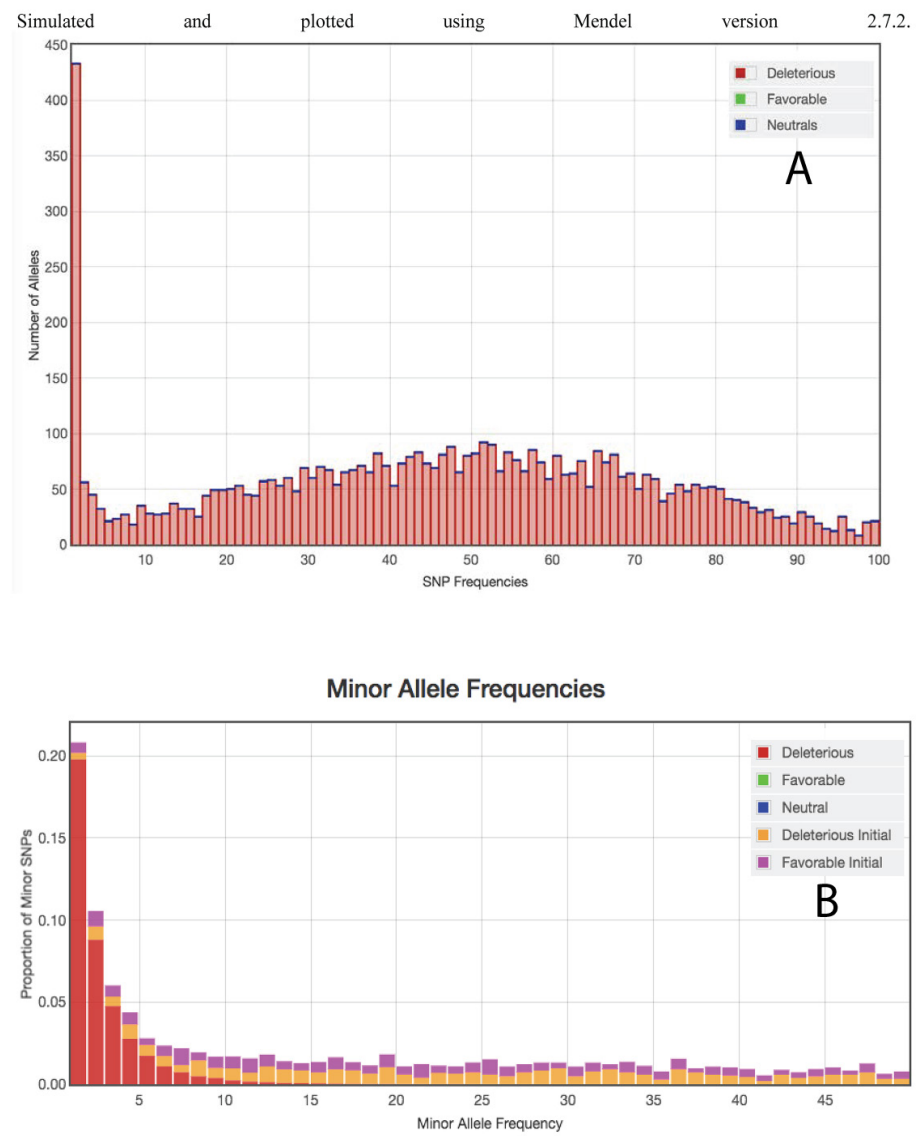

Figure 6a. A preliminary simulation combining both mutational and designed alleles (all designed alleles starting at 50\%). We performed this simulation using biblical parameters (an initial population of two, and ten generations later severe single generation bottleneck with 6 individuals), and a mixture of designed and created alleles. This run is essentially a combination of Figure $2 \mathrm{~d}$ and Figure $3 \mathrm{c}$. Note how different this distribution is from the actual allele distribution (Figure 1a). The spike of alleles on the far left is due to the continuous accumulation of mutational alleles, most of which remain rare throughout the 200 generations of the experiment. The broad bulge centered on 0.50 represents the designed alleles - all of which started at a frequency of $50 \%$. Note the problematic "gap" in the distribution, in the range of 3-20\%. Mendel has plotted allele frequencies from $1-100 \%$. Simulated and plotted using Mendel version 2.7.2.

Figure 6b. This is a heterozygous Adam and Eve simulation. A simulation combining both mutational and initially designed alleles (with all designed alleles starting at $25 \%$ ). We performed this simulation using biblical parameters and a mixture of initial designed alleles and accumulating mutational alleles (case ff824f). There were 296,700 initial designed allele pairs (purple alleles are less favored and gold alleles are more favored). The accumulated mutational alleles are shown in red. Note how similar this distribution is compared to the actual allele distribution (Figure 1a, Figure 8). Mendel has plotted allele frequencies from 1-50\%. Simulated and plotted using new Mendel-Go version.

It is easy to envision a great deal of genetic diversity being frontloaded into the genomes of Adam and Eve. However, it is more difficult to understand how such designed allelic variation could result in allele distribution patterns that are strongly skewed toward low-frequency alleles, and hence line up with the actually observed human allele distribution. We have simulated evolutionary populations in deep time (Figure 2b). We have simulated 
evolutionary Adam and Eve scenarios, where Adam and Eve derive from an evolutionary population, constituting an extreme single-generation bottleneck, and then in just 200 generations give rise to modern humanity. We have simulated biblical populations where Adam and Eve are created miraculously as the first human couple just 200 generations ago, being massively heterozygous by design (Figures $5 \mathrm{~b}$ ), with a subsequent population bottleneck at the time of Noah. We have simulated biblical populations where Adam and Eve begin as the first couple just 200 generations ago, having their millions of gametogonia created genetically distinct - such that their sperm and egg cells would represent a very large gene pool (Figure 7). Figure 8 shows the distributions of Figures 1a, $2 \mathrm{~b}, 2 \mathrm{c}, 6 \mathrm{~b}$, and 7 , side-by-side. It is visually obvious that the three types of Adam and Eve simulations closely aligned with chr22, while the evolutionary simulation was most discordant. This is also shown quantitatively in Table 2, which shows which distributions were closest to the actually observed distribution. All three of our Adam and Eve simulations could yield allele distributions very similar to the actually observed allele distribution. Again, it was our evolutionary simulation that was most discordant with the actually observed allele distribution.

All of the types of simulations listed above can be further fine-tuned to yield allele distributions that better match the actually observed allele frequency distribution. Both evolutionists and creationists can invoke hypothetical mechanisms to bring their simulated curves into closer alignment with the actual data. The evolutionary simulation (Figure2b) indicates a distinctly softer "bend" in the distribution, compared to the actually observed distribution. In order to reconcile evolutionary simulations with the real data, evolutionists need to invoke a long-term population bottleneck in the distant past (all bottlenecks seem to tighten the bend). Similarly, in order to match the actually observed allele distribution, our biblical simulations clearly require at least one severe population size constriction. While both the evolutionary and biblical models require genetic bottlenecking, for the evolutionary model to invoke a bottleneck is entirely post hoc, while for the creation model bottlenecking is inherent and integral.

Most geneticists assume allele frequency distributions arise primarily by random accumulation of mutations and random genetic drift, both of which require deep time. Given that perspective, it would seem impossible for two people to give rise to modern humanity in just a few hundred generations. However, from a creation perspective, we see many SNPs not as mutational alleles but as created alleles. This greatly reduces the time needed to generate the observed number of SNPs. Likewise, there are demographic forces that can cause allele frequencies to shift much faster than classic random genetic drift can accomplish. We suggest there is a real need for a more realistic model of genetic drift and allele frequency change. We are convinced that a more realistic understanding of how alleles change (apart from mere gametic sampling) will greatly reduce the time needed to generate the actually observed allele distribution.

Drift is thought to happen almost exclusively due to tiny sampling fluctuations in the gametic gene pool, generation after generation. This is a diffusion model - very slow, very steady, very clocklike. It is an ever-present, entropic dissipation. Not only is the

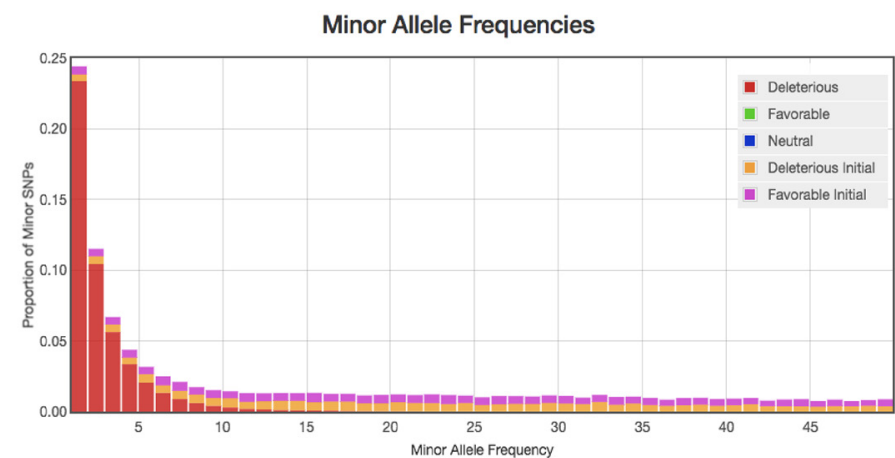

Figure 7. This is a designed gametes simulation. The population begins with 50 offspring of Adam and Eve who were derived from 100 of Adam and Eve's designed gametes (gametegonia) (case w35b49). This initial population grows for 9 generations, followed by a biblical bottleneck (3 reproducing couples), and then regrowth up to 1000 individuals. The simulation was halted in the $200^{\text {th }}$ generation. Mutational alleles are shown in red, while designed alleles are purple (un-favored alleles) or gold (favored alleles). All alleles were near-neutral. The plotted allele frequencies are from $1-50 \%$. Simulated and plotted using new Mendel-Go version.

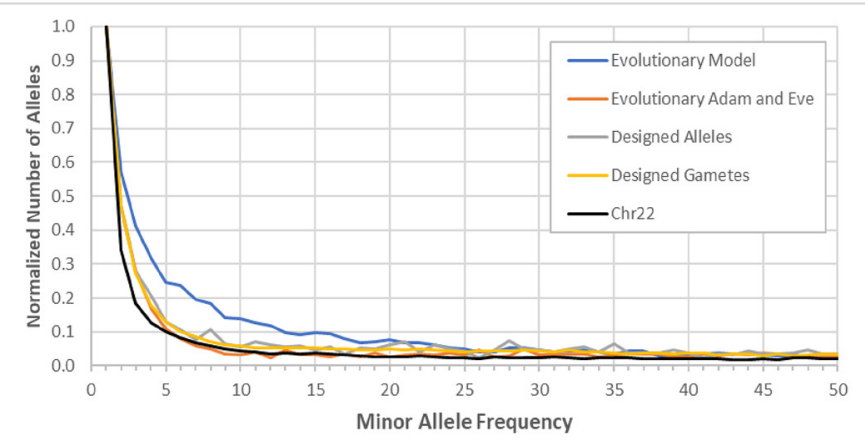

Figure 8. The normalized distributions of Figures 1a (chromosome 22), 2b (Evolutionary Model), 2c (Evolutionary Adam and Eve Model), 6b (Designed Alleles Model), and 7 (Designed Gametes Model), plotted for purposes of comparison. Clearly, a number of different biblical models align surprisingly well with the actually observed allele frequency data.

standard model of genetic drift extremely slow and weak, it is extremely unrealistic biologically. It assumes that; 1) there is no natural selection happening; and 2) there is perfect random mating (no sub-populations). Both of these assumptions are known to be profoundly wrong.

We suggest that for higher organisms any real global population is always being subjected to strong demographic forces that are much more powerful than genetic drift, causing allele frequency patterns to change much faster than has ever been simulated. If we consider recent human history, it is clear that the primary cause of changing allele patterns has not been due to random drift but has been due to many other demographic factors. For example, the human population has continuously experienced dramatic changes in composition due to war, conquest, disease, technology, etc. In the recent past, the European population exploded as colonialism went global. Multiple factors caused Native American populations to collapse. At present European, Japanese, and Korean populations are shrinking. At the same time the people of India, many parts of 
Africa, and many Muslim populations are exploding. Arguable all these types of genetic change have been going on for as long as man has existed. Logically, these forces should cause "accelerated drift" (i.e., accelerated allele frequency change). If these forces are ignored, genetic simulations will consistently indicate that populations are older than they really are, and that populations can only change extremely slowly. A major new paper has just been released that demonstrates rapid and massive demographic shifts in the early human population (Narasimhan et al. 2018). This study, involving 92 scientists, clearly shows the historical reality of massive global demographic shifts. All such major demographic shifts should effective accelerate allele spreading.

A simple illustration of how genetic drift might really be operating at a much higher rate, can be seen when we consider a colored liquid carefully added to water. If there is very little initial mixing, the added solution will diffuse very slowly and at a constant rate. But if there is any type stirring, the rate of mixing is much faster and the exact rate becomes unpredictable. Another illustration would be trying to study ocean chemistry or marine biology based on diffusion alone, without taking into account ocean currents. It should be obvious that there are numerous demographic forces that act to "stir" the gene pool of any global population. We propose the term demographic stirring to describe this phenomenon, and we suggest that it is ubiquitous in nature. Any type of demographic stirring should greatly accelerate the rate of genetic drift and should eclipse that special type of drift that is simply diffusion/ sampling error. We suggest all future population modeling and simulation should take into consideration demographic stirring. People doing genetic simulations (including the authors of this paper), have failed to include these important demographic forces in their models for the simple reason that these factors are complicated and challenging to realistically simulate. To take into account demographic stirring, population models and simulations need some type of correction factor.

All the simulation experiments recorded in this paper employed a maximal population size of 1000 individuals, instead of the conventionally assumed historical human population size of 10,000 individuals. Our smaller populations help compensate for the complete absence of any natural "demographic stirring" in our simulations. We observe that this "correction factor" very effectively accelerates allele spreading, yielding the distributions we show in Figure 8. It is important to realize that while the rate of classical genetic drift is almost entirely a function of population size, demographic stirring is not directly tied to population size. Therefore, in larger global populations, classical genetic drift is essentially irrelevant, and the only meaningful factors that change allele frequencies are natural selection and other types of demographic stirring.

A major limitation of numerical simulation is that it lacks the ability to model extremely large populations. In particular, we cannot model the billions of very rare alleles that are now accumulating in our very large population. These nearly countless rare alleles are the un-plotted alleles in the "invisible bin" (frequencies $0-1 \%$ ) of our histograms and linear graphs. While it is a practical necessity to ignore such alleles in our plots, we cannot ignore them in our thinking. Historical rapid lineage expansions that have happened at the expense of other sub-populations (Narasimhan et al. 2018), should pull many alleles out of the invisible bin and into higher frequency bins. Our simulations fail to model the vast number of rare alleles that would accumulate as the human population grew rapidly from thousands of people to billions of people. Realistically, any type of demographic stirring would draw large numbers of SNPs into the $1-99 \%$ frequency range, helping to explain the large number of actually observed human polymorphisms. Therefore, there is an enormous reservoir of SNPs in the actual human population that Mendel is neither currently simulating or plotting.

We acknowledge that reducing population size is not a perfect correction factor - but it seems to us better than entirely ignoring the numerous major demographic factors such as natural selection and lineage expansions. Ideally, all population models and simulations should eventually take into consideration some degree of demographic stirring and should include some type of demographic correction factor to achieve greater biological realism. Despite the complication associated with substituting reduced population size for demographic stirring, we are very encouraged by what our simulations show. We summarize below what we have learned about the three primary mechanisms that help us reconcile a literal Adam and Eve with the observed human allele distribution.

\section{Adam and Eve were created heterozygous, followed by population constrictions that accelerated genetic drift.}

Our preliminary simulations of a heterozygous Adam and Eve were over-simplified (Figures 3a, 3b, 3c, 4a, 4b, 6a) and yielded distributions different from the actually observed allele frequency distribution (Figure 1a). When designed alleles were combined with newly arising mutations, the designed alleles had a humped distribution along the $\mathrm{x}$-axis, while the mutational alleles had a nearly vertical distribution, with almost all mutational alleles being squeezed into the first bin on the far left of the histogram (Figure 6a). Based upon our preliminary simulations, it seemed problematic for us to reconcile the designed diversity model to the observed allele frequency distribution.

We found that our results began to approximate the modern allele distributions when we added other key elements to our formula. This included several instances of reduced or constrained population size, and more than one initial allele frequency.

Most importantly, we required accelerated genetic drift, which is essential for filling the allele distribution "gap" in the range of $3-20 \%$. It is generally assumed that accelerated genetic drift only happens when a population is relatively small. As soon as the population size reaches 1,000 or more, classical genetic drift grinds to a near standstill (Carter and Powell 2016). Substantial allele spreading required that early in the simulation there must be at least one episode where the population size is very small for a number of generations. Fortunately, the biblical model provides two and perhaps three such episodes: a) the tiny initial population in Eden, consisting of just two people; $b$ ) the tiny post-flood population of just six reproducing adults; and c) a possible stall in population growth among the emerging tribes, following the dispersion out of Babel (which may well have been chaotic/violent). We might have simulated a single but more prolonged population bottleneck 
to accomplish the same thing, however we sought to be consistent with the biblical account, which seems to require moderately rapid population growth after Adam and Eve, a single-generation bottleneck at the time of the Flood, and moderately rapid population rebound after the flood (Carter and Hardy 2015).

When we combined mutational alleles with designed alleles (starting at $25 \%$ ), with the two primary bottlenecks, we saw that the designed alleles substantially drifted toward lower allele frequencies, while the mutational alleles drifted substantially toward higher allele frequencies, effectively filling the gap (Figure $6 b)$.

When we added a blend of two or more types of designed allele pairs (for example, one type starting with an initial allele frequency of $25 \%$, another type starting with an initial allele frequency of $50 \%$ ), we found our distributions could be fine-tuned to align with the actually observed allele distribution (Figure 7).

In summary, modeling a heterozygous Adam and Eve brings us a very long way toward reconciling a literal Adam and Eve with the observed allele frequencies. In this model, the primary factors that shape the allele frequency distribution included: a) the number and duration of bottlenecks; b) the ratio of the designed alleles in different frequency classes; and c) the ratio of designed alleles versus mutational alleles. By modulating these variables, we were able to discover parameter settings that produce allele frequency distributions that plot as smooth curves and closely approximate the actually observed biological allele frequency distributions. We may be able to enhance the current distribution further by: a) adjusting the ratio of mutational alleles versus designed alleles; b) simulating a growth pause after the Babel event; and c) simulating an episode of accelerated mutation accumulation in the first $25-50$ generations after the flood.

2. Adam and Eve were created with internal designed diversity, combined with various demographic forces other than classical genetic drift.

We believe that we must eventually factor in forces such as selective sweeps and differential sub-population expansions to make our simulations more biologically realistic.

Genetic drift is really just a type of diffusion and is quite impotent in changing allele frequencies except in very small populations or over very deep time. It is a passive and slow process. Most drift models (including ours) assume all alleles are neutral, that there is essentially no selection, and that the global population has perfectly random mating. Yet there are various other forces that are much more effective in shifting allele frequencies, and in much less time. These other factors consist of numerous active processes such as selective sweeps, migration/invasion, explosive lineage expansions, lineage extinctions, and other fast-acting population events.

We used selective sweeps as just one example to illustrate non-drift alternatives that can change allele frequencies rapidly (Figure 5). A series of powerful selective sweeps can clearly help fill "the gap". One reason why designed diversity makes so much sense is that it enables very rapid adaptation to local conditions. The biblical mandate to "fill the earth" (i.e., fill all the environmental niches in the earth), seems to imply rapid adaptive population fragmentation.
Therefore, it would be reasonable to have a limited class of designed alleles on hand that could rapidly respond to natural selection. Designed alleles represent the most effective and the most rapid way to cause selection-driven adaptation. This is because no extended "waiting time" is required. All the required genetic variants are present from the first generation, and are already present at high frequency, enabling very rapid selective progress. There is no need to wait for just the right set of mutations to arise serendipitously and then slowly move from allelic near-extinction to allelic fixation. Furthermore, such designed variants would already exist as fully functional linkage sets at high frequency. This amplifies the rate of selective progress and so allows for extremely rapid adaptation. Given a pair of high-impact alleles, one allele will typically be more adaptive in one habitat than the other, so the frequency of that allele would increase rapidly toward fixation in that habitat. At the same time, in that habitat the corresponding minor allele would be moving toward a frequency of zero. As these high-impact pairs are driven toward the far left or far right of our plots, they will carry with them countless nearly-neutral variants that happen to be linked. This is what is called a "selective sweep". Those variants that will be carried along will include both designed alleles and mutational alleles. In less than 200 generations we observe smooth allele distributions when we simulated this scenario (Figure 5).

Given only mutational alleles, selective sweeps would be expected to be very rare. This is because beneficial mutations are very rare, and because mutations that are strongly-beneficial are vanishingly rare. However, given designed alleles, selective sweeps (and all other types of adaptive selection) should be very common, and should respond to selection very rapidly. This is because designed alleles would be very abundant from the first generation (no waiting time), they would be created at relatively high frequencies, and for every designed allele pair both variants would have a designed purpose, one favored over the other, depending on habitat. Lastly, designed alleles of this type would naturally be designed to work in coordination each other, constituting functional linkage blocks, and constituting desirable poly-genic (quantitative) traits.

We used selective sweeps as our example because they are rapid demographic shifts that can be easily demonstrated using numerical simulation. However, we are not yet able to simulate other important demographic factors that could very rapidly change allele frequencies. Looking backwards in human history, we see a long series of explosive human expansions, along with genocides and shrinkages. It is obvious that all people living today are the descendants of the lucky lineages that survived, as the vast majority of all lineages go extinct (Helgason et al. 2003; Rohde et al. 2004). Therefore, the frequencies of many human alleles must have diminished over time, even as the alleles of other historical populations surged forward. These types of population dynamics could rapidly amplify many of the countless un-plotted rare mutational alleles, moving them from the invisible $0-1 \%$ histogram bin into the $1-15 \%$ histogram bins.

We have primarily compared our simulated results with the actual allele distributions of the autosomal chr22. But we also generated the actual allele distributions of chrY and chrM. These distributions are striking in that these two chromosomes are very different in their nature, yet both of their distributions are very similar. They both 
have very few high-frequency polymorphisms. Overwhelmingly, the polymorphic alleles have very low frequencies. This is consistent with the Heterozygous Adam and Eve Model. These two very special chromosomes would have existed in Eden, not in four copies, but as single ancestral copies. Thus, they would have had zero designed alleles, and all the observed polymorphisms would be the result of newly accumulating mutations. We suggest that if we could strip all the designed alleles out of the rest of the genome, all human chromosomes would have allele distributions very similar to chrY and chrM. These chrY and chrM distributions seem to reflect very young chromosomes. It appears that there has been too little time for these chromosomes to accumulate very many mutational alleles and have them drift to higher frequencies.

\section{Adam and Eve's created gametogonia contained designed diversity.}

There could have been a vast number of created gametogonia within Adam and Eve. Each gametogonium could have been genetically unique. Every gamete in Eden could have been designed with its own unique set of alleles and its own unique linkage patterns. That original population of reproductive cells could have represented a "gene pool" comparable to a large human population.

We have used numerical simulation to illustrate how designed gametes might give rise to modern allele frequencies. Our designed gametes simulations model a population that begins with 50 offspring of Adam and Eve, who were derived from 100 of Adam and Eve's designed gametes (gametegonia). This initial population was designed with 989,000 or more allele pairs, with the designed alleles initially having a spectrum of frequencies. The population was allowed to grow for 9 generations, followed by a biblical bottleneck ( 3 reproducing couples), and then regrow up to 1000 individuals. The simulation was halted in the $200^{\text {th }}$ generation. The subsequent allele frequency spectrum is shown in Figure 7. Each designed allele pair had a purple (un-favored) allele, and a gold (favored) allele. All alleles were nearly-neutral in fitness effect. The plotted allele frequencies were from $1-50 \%$. The designed gametes allele distribution was very similar to the actually observed distribution.

Under the Designed Gametes Model, functional variants would have logically been organized into functional linkage blocks. This would not just create a vast amount of diversity, it would also create a vast number of fully functional and fully integrated linkage blocks within the genome. This model seems to most effectively refute the audacious claim that "There is no way...". This model is not only consistent with what is observed, it would appear to be very hard to falsify. More than a strong counter-argument, this model seems to appropriately reflect the glory of God, the beauty and elegance of His design, and the nature of His providential planning. This biblical model is not only reasonable, it has inspired the authors to reflect, with wonder, upon what the human race might have been like if there had never been a Fall.

In summary, our research indicates that it is reasonable that two miraculously created people, if endowed with appropriate designed genetic diversity, could have given rise to allele distributions similar to what we see today. Indeed, given a miraculous creation, it would only seem logical for the Creator to pre-program designed diversity into the genomes of the first couple. In retrospect, it would seem remarkable if $\mathrm{He}$ did not include beneficial types of variation. Our research indicates that after the miraculous creation event, there could be several ways that natural processes (such as accelerated genetic drift, selective sweeps, and sub-population surges), could help give rise to the specific allele distributions observed today.

We began to investigate these issues more than a decade ago. Although the issues are complex, it is now very clear that the theistic evolutionist's claim that "there is no way..." (that a literal Adam and Eve could ever give rise to our current allele distribution) was seriously over-reaching. In light of the current study, that claim appears to be incorrect. Given what is at stake-the authority of Scripture and the faith of millions of people-this militant attack on the historical Adam and Eve that is coming from within the church appears to be reckless and destructive. We exhort our Christian brethren who have been so vigorously arguing against a literal Adam and Eve to very carefully consider the possibility that they may be mistaken, and to prayerfully consider the possibility that they are undermining the faith of millions of souls.

\section{CONCLUSIONS}

In this paper we have used logic and numerical simulation to show that there are several Designed Diversity mechanisms that can reconcile a literal Adam and Eve with the allele frequency distribution now seen in the human population. These genetic mechanisms include: 1) designed diversity within Adam and Eve's four sets of chromosomes followed by accelerated genetic drift associated with multiple population constrictions; 2) as above, combined with more powerful demographic forces such as selective sweeps, lineage extinctions, and differential subpopulation expansions; and 3) designed diversity within Adam and Eve's originally created gametogonia. Together, these various genetic mechanisms seem to falsify the claim that there is "no way" that two people could give rise to the human allele distribution that we see today. The designed gametes model appears to be especially robust, and in our opinion is even elegant. It seems to be the best explanation for how Adam and Eve might have simultaneously given rise to our current human allele patterns and our current linkage patterns. Future research will examine the concept of "demographic stirring" and how it may accelerate genetic drift.

Given the premise of a miraculously created Adam and Eve, the most coherent, powerful, and compelling explanation for most of the genetic diversity found within the human race is "designed diversity". This is especially true when we consider the various forms of human beauty and the various forms of human gifts and talents. Human traits of this type cannot rationally be attributed to Darwinian mutation/selection. In addition, designed diversity appears to have enabled rapid human adaptation after the flood.

\section{ACKNOWLEDGEMENTS}

We gratefully thank Stephen Lee for advice regarding statistical analysis.

\section{REFERENCES}

1000 Genomes Project Consortium. 2015. A global reference for human genetic variation. Nature 52:68-74.

Baharian, S., and S. Gravel. 2018. On the decidability of population size histories from finite allele frequency spectra. Theoretical Population 


\section{Biology 120:42-51.}

Baumgardner, J., J. Sanford, W. Brewer, P. Gibson, and W. ReMine. 2008. Mendel's Accountant: a new population genetics simulation tool for studying mutation and natural selection. In Proceedings of the Sixth International Conference on Creationism, ed. A.A. Snelling, pp. 8798. Pittsburgh, Pennsylvania: Creation Science Fellowship and Dallas, Texas: Institute for Creation Research.

Baumgardner, J., W. Brewer, and J. Sanford. 2013. Can synergistic epistasis halt mutation accumulation? Results from numerical simulation. In Biological Information-New Perspectives, eds. R.J. Marks II, M.J. Behe, W.A. Dembski, B. Gordon, and J.C. Sanford, pp. 312-337. Singapore: World Scientific.

Brewer, W., J. Baumgardner, and J. Sanford. 2013. Using numerical simulation to test the "mutation-count" hypothesis. In Biological Information-New Perspectives, eds. R.J. Marks II, M.J. Behe, W.A. Dembski, B. Gordon, and J.C. Sanford, pp. 298-311. Singapore: World Scientific.

Brewer, W., J. Baumgardner, P. Gibson, and J. Sanford. 2013. Can purifying natural selection preserve biological information? In Biological Information-New Perspectives, eds. R.J. Marks II, M.J. Behe, W.A. Dembski, B. Gordon, and J.C. Sanford, pp. 232-263. Singapore: World Scientific

Brewer, W., F.D. Smith, and J.C. Sanford. 2013. Information loss: Potential for accelerating natural genetic attenuation of RNA viruses. In Biological Information - New Perspectives, eds. R.J. Marks II, M.J. Behe, W.A. Dembski, B. Gordon, and J.C. Sanford, pp. 369-384. Singapore: World Scientific

Carter, R.W. 2007. Mitochondrial diversity within modern human populations. Nucleic Acids Research 35, no. 9:3039-3045.

Carter, R., D. Criswell, and J.C. Sanford. 2008. The "Eve" mitochondrial consensus sequence. In Proceedings of the Sixth International Conference on Creationism, ed. A.A. Snelling, pp.111-116. Pittsburgh, Pennsylvania: Creation Science Fellowship and Dallas, Texas: Institute for Creation Research.

Carter R., and M. Powell. 2016. The genetic effects of the population bottleneck associated with the Genesis Flood. Journal of Creation 30, no. 2:102-111.

Carter, R.C., and J.C. Sanford. 2012. A new look at an old virus: patterns of mutation accumulation in the human H1N1 influenza virus since 1918. Theoretical Biology and Medical Modeling 9:42.

Carter, R.W. 2014. More evidence for the reality of genetic entropy. Journal of Creation 28, no. 1:16-17.

Carter, R. and C. Hardy. 2015. Modelling biblical human population growth. Journal of Creation 29, no. 1:72-79.

Carter, R. 2015. Finally setting the record straight - Christian views on the historicity of Adam through the ages. Journal of Creation 29, no. 2:36-40.

Carter, R.W. 2017. Reading evolution into the Scriptures: a review of Adam and the Genome: Reading scripture after genetic science (Dennis R. Venema and Scot McKnight). Journal of Creation 31, no. 2:41-46.

Carter, R.W. 2018. Effective population sizes and loss of diversity during the Flood bottleneck. Journal of Creation 32, no. 2:124-127..

Carter, R.W., S.S. Lee, and J.C. Sanford. 2018. An overview of the independent histories of the human $\mathrm{Y}$ chromosome and the human mitochondrial chromosome. In Proceedings of the Eighth International Conference on Creationism, ed. J.H. Whitmore, pp. 133-151. Pittsburgh, Pennsylvania: Creation Science Fellowship.
Cordova, S.T., and J.C. Sanford. 2017. Nylonase genes and proteins distribution, conservation, and possible origins. Download available at http://vixra.org/pdf/1708.0370v1.pdf

Dirks, P., E.M. Roberts, H. Hilbert-Wolf, J.D. Kramers, J. Hawks, A. Dosseto, M. Duval, M. Elliott, M. Evans, R. Grün, J. Hellstrom, A. Herries, R. Joannes-Boyau, T.V. Makhubela, C.J. Placzek, J. Robbins, C. Spandler, J. Wiersma, J. Woodhead, and L.R. Berger. 2017. The age of Homo naledi and associated sediments in the Rising Star Cave, South Africa. eLife 6:e24231.

Diroma, M.A., C. Calabrese, D. Simone, M. Santorsola, F.M. Calabrese, G. Gasparre, M. Attimonelli. 2014. Extraction and annotation of human mitochondrial genomes from 1000 Genomes Whole Exome Sequencing data. BMC Genomics 15(Suppl 3):S2.

Faulk, D. 2004. Coming to peace with science: Bridging the worlds between faith and biology. Intervarsity Press, Downers Grove, IL.

Gauger, A. Does science rule out a first human pair? Geneticist Richard Buggs says no. October 3, 2017. Retrieved March 22, 2018 from http:// www.evolutionnews.org/2017/10/does-science-rule-out-a-first-humanpair-geneticist-richard-buggs-says-no.

Gibson, P., J. Baumgardner, W. Brewer, and J. Sanford. 2013. Can biological information be sustained by purifying natural selection? In Biological Information-New Perspectives, eds. R.J. Marks II, M.J. Behe, W.A. Dembski, B. Gordon, and J.C. Sanford, pp. 232-263. Singapore: World Scientific.

Gierlinski G.D., G. Niedźwiedzki, M.G. Lockley, A. Athanassiou, C. Fassoulas, Z. Dubicka, A. Boczarowski, M.R. Bennett, and P.E. Ahlberg. 2017. Possible hominin footprints from the late Miocene (c. 5.7 Ma) of Crete? Proceedings of the Geologists' Association 621:1-14.

Gurdasani, D., T. Carstensen, F. Tekola-Ayele, L. Pagani, I. Tachmazidou, K. Hatzikotoulas, S. Karthikeyan, L. Iles, M.O. Pollard, A. Choudhury, G.R.S. Ritchie, Y. Xue, J. Asimit, R.N. Nsubuga, E.H. Young, C. Pomilla, K. Kivinen, K. Rockett, A. Kamali, A.P. Doumatey, G. Asiki, J. Seeley, F. Sisay-Joof, M. Jallow, S. Tollman, E. Mekonnen, R. Ekong, T. Oljira, N. Bradman, K. Bojang, M. Ramsay, A. Adeyemo, E. Bekele, A. Motala, S.A. Norris, F. Pirie, P. Kaleebu, D. Kwiatkowski, C. TylerSmith, C. Rotimi, E. Zeggini, and M.S. Sandhu. 2014. The African Genome Variation Project shapes medical genetics in Africa. Nature 517, no. 7534:327-332.

Harpak, A., A. Bhaskar, and J.K. Pritchard. 2016. Mutation rate variation is a primary determinant of the distribution of allele frequencies in humans. PLoS Genetics 12, no. 12:e1006489.

Helgason, A., B. Hrafnkelsson, J.R. Gulcher, R. Ward, and K. Stefánsson. 2003. A populationwide coalescent analysis of Icelandic matrilineal and patrilineal genealogies: evidence for a faster evolutionary rate of mtDNA lineages than y chromosomes. American Journal of Human Genetics 72:1370-1388.

Holen S.R., T.A. Deméré, D.C. Fisher, R. Fullagar, J.B. Paces, G.T. Jefferson, J.M. Beeton, R.W. Cerutti, A.N. Rountrey, L. Vescera, and K.A. Holen. 2017. A 130,000-year-old archaeological site in southern California, USA. Nature 544:479-483.

Hössjer O., A. Gauger, and C. Reeves. 2016a. Genetic modeling of human history part 1: Comparison of common descent and unique origin approaches. BIOComplexity 3:1-15. doi:10.5048/BIO-C.2016.3.

Hössjer O., A. Gauger, and C. Reeves. 2016b. Genetic modeling of human history part 2: A unique origin algorithm. BIO-Complexity 4:1-36. doi:10.5048/BIO-C.2016.4.

Hublin, J., A. Ben-Ncer, S.E. Bailey, S.E. Freidline, S. Neubauer, M.M. Skinner, I. Bergmann, A. Le Cabec, S. Benazzi, K. Harvati, and P. Gunz. 
2017. New fossils from Jebel Iroud, Morocco and the pan-African origin of Homo sapiens. Nature 546:289-292.

Jeanson, N.T., and J. Lisle. 2016. On the origin of eukaryotic species' genotypic and phenotypic diversity. Answers Research Journal 9:81122.

Jeanson, N., and J. Tomkins. 2017. Genetics confirms the recent supernatural creation of Adam and Eve. In Searching for Adam, ed. T. Mortenson, pp. 307-312. Green Forest, Arkansas: Master Books.

Kolbe, J.J., M. Leal, T.W. Schoener, D.A. Spiller, and J.B. Losos. 2012. Founder effects persist despite adaptive differentiation: A field experiment with lizards. Science 335, no. 6072:1086-1089.

Levy, S., G. Sutton, P.C. Ng, L. Feuk, A.L. Halpern, B.P. Walenz, N. Axelrod, J. Huang, E.F. Kirkness, G. Denisov, Y. Lin, J.R. MacDonald, A.W.C. Pang, M. Shago, T.B. Stockwell, A. Tsiamouri, V. Bafna, V. Bansal, S.A. Kravitz, D.A. Busam, K.Y. Beeson, T.C. McIntosh, K.A. Remington, J.F. Abril, J. Gill, j. Borman, Y-.H. Rogers, M.E. Frazier, S.W. Scherer, R.L. Strausberg, and J.C.Venter. 2007. The diploid genome sequence of an individual human. PLoS Biology 5, no. 10:e254.

Lightner, J. 2016. Towards a creationary view of why speciation occurs. Journal of Creation 30(1):70-75.

Marks II, R.J., M.J. Behe, W.A. Dembski, B. Gordon, and J.C. Sanford (eds). 2013. Biological Information-New Perspectives. World Scientific, Singapore.

Matute, D.R. 2013. The role of founder effects on the evolution of reproductive isolation. Journal of Evolutionary Biology 26:2299-2311.

Matute, D.R. 2014. The magnitude of behavioral isolation is affected by characteristics of the mating community. Ecology and Evolution 4, no. 14: 2945-2956.

Mortenson, T. (ed). 2016. Searching for Adam. Green Forest, Arkansas: Master Books,

Myers, S., C. Fefferman, and N. Patterson. 2008. Can one learn history from the allelic spectrum? Theoretical Population Biology 73:342-248

Narasimhan, V.M., N.J. Patterson, P. Moorjani, I. Lazaridis, L. Mark, S. Mallick, N. Rohland, R. Bernardos, A.M. Kim, N. Nakatsuka, I. Olalde, A. Coppa, J. Mallory, V. Moiseyev, J. Monge, L.M. Olivieri, N.Adamski, N. Broomandkhoshbacht, F. Candilio, O. Cheronet, B.J. Culleton, M. Ferry, D. Fernandes, B. Gamarra, D. Gaudio, M. Hajdinjak, E. Harney, T.K. Harper, D. Keating, A.-M. Lawson, M. Michel, M. Novak, J. Oppenheimer, N. Rai, K. Sirak, V. Slon, K. Stewardson, Z. Zhang, G. Akhatov, A.N. Bagashev, B. Baitanayev, G.L. Bonora, T. Chikisheva, A. Derevianko, E. Dmitry, K. Douka, N. Dubova, A. Epimakhov, S. Freilich, D. Fuller, A. Goryachev, A. Gromov, B. Hanks, M.Judd, E. Kazizov, A. Khokhlov, E. Kitov, E. Kupriyanova, P. Kuznetsov, D. Luiselli, F.Maksudov, C. Meiklejohn, D.C. Merrett, R. Micheli, O. Mochalov, Z. Muhammed, S. Mustafakulov, A. Nayak, R.M. Petrovna, D. Pettner, R. Potts, D. Razhev, S. Sarno, K. Sikhymbaevae, S.M. Slepchenko, N. Stepanova, S. Svyatko, S. Vasilyev, M. Vidale, D.Voyakin, A. Yermolayeva, A. Zubova, V.S Shinde, C. LaluezaFox, M. Meyer, D. Anthony, N. Boivin, K. Thangaraj, D. Kennett, M. Frachetti, R. Pinhasi, and D. Reich. 2018. Genomic formation of south and central Asia. bioRxiv. doi: https://doi.org/10.1101/292581.

Nei, W., T. Maruyama, and R. Chakraborty. 1975. The bottleneck effect and genetic variability of populations. Evolution 29, no. 1:1-10.

Nelson, C.W., and Sanford, J. 2013. Computational evolution experiments reveal a net loss of genetic information despite selection. In Biological Information-New Perspectives, eds. R.J. Marks II, M.J. Behe, W.A. Dembski, B. Gordon, and J.C. Sanford, pp. 338-368 Singapore:World Scientific.
Pennisi, E. 2012. ENCODE Project writes eulogy for junk DNA. Science 337, no. 6099:1159-1161.

Rohde, D.L.T, S. Olsen, and J.T. Chang. 2004. Modelling the recent common ancestry of all living humans. Nature 431:562-566.

Rupe, C., and J.C. Sanford. 2017. Contested Bones. Waterloo, New York: FMS Publications.

Rupe, C.L., and J.C. Sanford. 2013. Using numerical dimulation to better understand fixation rates, and establishment of a new principle: Haldane's Ratchet. In Proceedings of the Seventh International Conference on Creationism, ed. M.F. Horstemeyer. Pittsburgh, Pennsylvania: Creation Science Fellowship.

Sanford, J.C., J. Baumgardner, W. Brewer, P. Gibson, and W, ReMine. 2007. Using computer simulation to understand mutation accumulation dynamics and genetic load. In International Conference on Computational Science, eds. Y. Shi, G.D. van Albada, J. Dongarra, P.M.A. Sloot, pp. 386-392. [ICCS 2007, Part II. Lecture Notes in Computer Science, vol 4488]. Berlin, Heidelberg: Springer-Verlag.

Sanford, J., J. Baumgardner, W. Brewer, P. Gibson, and W. ReMine. 2007. Mendel's Accountant: A biologically realistic forward-time population genetics program. Scalable Computing: Practice and Experience 8, no. $2: 147-165$.

Sanford, J., J. Baumgardner, W. Brewer, P. Gibson, and W. ReMine. 2008. Using numerical simulation to test the validity of neo-Darwinian theory, in Proceedings of the Sixth International Conference on Creationism, ed. A.A. Snelling, pp. 165-175. Pittsburgh, Pennsylvania: Creation Science Fellowship and Dallas, Texas: Institute for Creation Research.

Sanford, J., and C. Nelson. 2012. The next step in understanding population dynamics: Comprehensive numerical simulation. In Studies in Population Genetics, ed. M.C. Fusté, pp. 117-135. Rijeka, Croatia: InTech.

Sanford, J., J. Baumgardner, and W. Brewer. 2013. Selection threshold severely constrains capture of beneficial mutations. In Biological Information-New Perspectives, eds. R.J. Marks II, M.J. Behe, W.A. Dembski, B. Gordon, and J.C. Sanford, pp. 264-297 Singapore: World Scientific.

Sanford, J.C. 2014. Genetic Entropy. FMS Publications, Waterloo, NY.

Sanford, J.C., and R. Carter. 2014. In light of genetics...Adam, Eve, and the creation/fall. Christian Apologetics Journal 12, no. 2:51-72.

Sanford, J., W. Brewer, F. Smith, and J. Baumgardner. 2015. The waiting time problem in a model hominin population. Theoretical Biology and Medical Modelling 12:18.

Sanford, J.C., and R. Carter. 2016a. God, family, and genetics - A biblical perspective, part 1: Genetic evidences supporting the divine origin of man and family. [Proceedings of the Symposium at Rome in 2015] "The Two Shall Become One": The Creation of Adam and Eve as the Foundation of the Church's Teaching on Holy Marriage. Human Life International.

Sanford, J.C., and R. Carter. 2016b. God, family, and genetics - A biblical perspective, part 2: Genetic evidences refuting the evolution of man and family. [Proceedings of the Symposium at Rome in 2015] "The Two Shall Become One": The Creation of Adam and Eve as the Foundation of the Church's Teaching on Holy Marriage. Human Life International.

Shaffner, S. 2017a. Can someone explain like I'm 5 yo, what's wrong with this refutation of Biologos? Retrieved May 15, 2018, from https:// discourse.biologos.org/t/can-someone-explain-like-im-5-yo-whatswrong-with-this-refutation-of-biologos/5657/16 [See also post \#126 on this same thread]. 
Shaffner, S., 2017b. Bottleneck: a forward genetic simulator for playing around with demographic bottlenecks. Retrieved May 15, 2018, from https:/github.com/glipsnort/bottleneck [This was the software used to generate the graphic in the discussion thread (Shaffner 2017a)].

Templeton, A.R. 2008. The reality and importance of founder speciation in evolution. BioEssays 30:470-479.

Terhorst, J., and Y.S. Song. 2015. Fundamental limits on the accuracy of demographic inference based on the sample frequency spectrum. Proceedings of the National Academy of Science (USA) 112, no. 25:7677-7682.

Tomkins, J. 2013. The human beta-globin pseudogene is non-variable and functional. Answers Research Journal 6:293-301.

Tomkins, J. 2015. Documented anomaly in recent versions of the BLASTN algorithm and a complete reanalysis of chimpanzee and human genomewide DNA similarity using Nucmer and LASTZ. Answers Research Journal 8:379-390.

Tomkins, J. 2017. Debunking the debunkers: a response to criticism and obfuscation regarding refutation of the human chromosome 2 fusion, Answers Research Journal 10:45-54.

VanDoodewaard, W. 2015. The Quest for the Historical Adam: Genesis, Hermeneutics, and Human Origins. Grand Rapids, Michigan: Reformation Heritage Books.

Venema, D.R. 2010. Genesis and the genome: genomics evidence for human-ape common ancestry and ancestral hominid population sizes. Perspectives on Science and Christian Faith 62, no. 3:166-178.

Venema, D.R., and S. McKnight. 2017. Adam and the Genome: Reading Scripture After Genetic Science. Brazos Press, Grand Rapids, MI.

Wood, T.C., and J.W. Francis. 2016. Genetics of Adam. In What Happened in the Garden?, ed. A. Chou, pp. 75-100. Grand Rapids, Michigan: Kregel.

Woodmorappe, J. 1996. Noah's Ark: a Feasibility Study [pp. 176-195]. Santee, California: Institute for Creation Research.

\section{THE AUTHORS}

As a Cornell University professor, John Sanford conducted genetic research for 37 years. This research resulted in more than 100 scientific publications, and several dozen patents. John is presently president of Logos Research Associates, and president of Feed My Sheep Foundation. John's most significant contributions to science have been: 1) primary inventor of the Biolistic Process (the "Gene Gun”); 2) author of the book Genetic Entropy; 3) co-developer of Mendel's Accountant (a comprehensive and biologically realistic numerical simulation of the mutation/selection process); 4) lead organizer/editor of the Cornell symposium and subsequently published proceedings entitled Biological Information--New Perspectives; and 5) coauthor of the book Contested Bones.
Dr. Robert W. Carter is a senior scientist and speaker for CMI-USA in Atlanta, Georgia. He is currently researching human genetics and other issues related to biblical creation. He obtained a BS in Applied Biology from the Georgia Institute of Technology in 1992. He then spent four years teaching high school biology, chemistry, physics and electronics before going to the University of Miami to obtain his PhD in Marine Biology.

Dr. Wes Brewer is a consultant for Computational Solutions LLC. He is a graduate of MIT and has a PhD in Computational Engineering. After spending a number of years developing computational hydrodynamic codes for the US Navy, he started his own consulting company in which he has worked with numerous companies and organizations around the world. He has spent almost a decade teaching computer science in both South and North Korea. Furthermore, he has worked on Mendel's Accountant team since 2005 and also has worked with Larry Vardiman/ICR on post ice age climate modeling.

Dr. John Baumgardner is vice-president of Logos Research Associates. He has a Ph.D. in geophysics and space physics from UCLA and worked at Los Alamos National Laboratory in computational physics research during most of his scientific career. Since the early 1980's he has undertaken most of the primary research undergirding the concept of catastrophic plate tectonics in connection with Noah's Flood. Beginning in 1997 he served on the Radioisotopes and the Age of the Earth (RATE) team that documented multiple independent lines of radioisotope evidence that the earth is thousands, not billions, of years old. Since 2005 he has been part of a small team that has developed Mendel's Accountant, a computer model for exploring key topics in population genetics relating to the origin and history of life. John currently is a senior research associate with Logos Research Associates based in Santa Ana, California, and teaches science apologetics courses at Southern California Seminary in the San Diego area.

Bruce Potter is a research associate for Feed My Sheep Foundation. He has degrees in computer science and mechanical engineering from Clarkson University. He has worked for IBM as a software engineer for 33 years, first on IBM's supercomputers, and more recently in cloud and edge computing. He has 2 patents, and 15 years of experience in developing open source code, including contributing to several open source projects.

Jonathan Potter is a research associate for Feed My Sheep Foundation. He has his BS and Masters degrees in Computer Science from Rochester Institute of Technology and has worked as a software engineer for six years. He specializes in web technologies and has contributed to multiple open source projects. 